\title{
Scalability of an Unstructured Grid Continuous Galerkin Based Hurricane Storm Surge Model
}

\author{
S. Tanaka ${ }^{1) *}$, S. Bunya ${ }^{2)}$, J.J. Westerink ${ }^{3)}$, C. Dawson ${ }^{4)}$, \\ R.A. Luettich, Jr. ${ }^{5)}$ \\ 1) Assistant Research Professor, \\ Environmental Fluid Dynamics Laboratories, \\ Department of Civil Engineering and Geological Sciences, \\ University of Notre Dame, Notre Dame, IN, U.S.A. \\ 2) Consultant, Science and Safety Policy Research Division, \\ Mitsubishi Research Institute, Inc., \\ Chiyoda-ku, Tokyo, Japan \\ 3) Professor, Environmental Fluid Dynamics Laboratories, \\ Department of Civil Engineering and Geological Sciences, \\ University of Notre Dame, Notre Dame, IN, U.S.A. \\ 4) Professor, Institute for Computational Engineering and Sciences, \\ The University of Texas at Austin, Austin, TX, U.S.A. \\ 5) Professor, Institute of Marine Sciences, \\ University of North Carolina at Chapel Hill, \\ Morehead City, NC, U.S.A.
}

Running Head:Scalability of Continuous Galerkin Based Hurricane Storm Surge Model

Submitted

January 20, 2010

In Revised Form

June 22, 2010

Journal of Scientific Computing

\footnotetext{
${ }^{*}$ Corresponding author: Seizo Tanaka, Environmental Fluid Dynamics Laboratories,

Department of Civil Engineering and Geological Sciences,

156 Fitzpatrick Hall, Notre Dame, IN, 46556, U.S.A.,

tel:(574)631-3864, fax:(574)631-9236,

e-mail: stanaka@nd.edu
} 


\begin{abstract}
This paper evaluates the parallel performance and scalability of an unstructured grid Shallow Water Equation (SWE) hurricane storm surge model. We use the ADCIRC model, which is based on the generalized wave continuity equation continuous Galerkin method, within a parallel computational framework based on domain decomposition and the MPI (Message Passing Interface) library. We measure the performance of the model run implicitly and explicitly on various grids. We analyze the performance as well as accurracy with various spatial and temporal discretizations. We improve the output writing performance by introducing sets of dedicated writer cores. Performance is measured on the Texas Advanced Computing Center Ranger machine. A high resolution 9,314,706 finite element node grid with 1s time steps can complete a day of real time hurricane storm surge simulation in less than 20 min of computer wall clock time, using 16,384 cores with sets of dedicated writer cores.
\end{abstract}

Keywords : parallel computing, scaling, shallow water equations, hurricane storm surge model, finite element method, forecasting 


\section{INTRODUCTION}

Hurricane storm surge is a low probabity but high impact disaster that can result in massive destruction of coastal infrastructure and significant loss of human life. Recent storms such as Katrina (2005), Rita (2005), Ike (2008) and Nargis (2008) have led to devastating damage. Hurricane storm surge and the resulting water levels and currents involve a wide range of processes as well as scales. Storm surge is regionally and locally generated in the coastal ocean, in inland waterbodies and over coastal floodplains and moves through rivers, inlets and channels. Storm surge is driven and affected by tides, river flows, wind waves, winds and atmospheric pressure.

The simulation of hurricane storm surge is a powerful tool used to evaluate risk, design hurricane protection systems, analyze the physics of storms, and plan evacuations [1]. These simulations must be fast and accurate in order to estimate the water level and current environment and assess the potential of risk and damage. In particular, within the realm of forcasting, two to four days of real time simulation must be completed within an hour of computer wall clock time in order to be useful to emergency planners. However, realistic solutions require the use of high resolution computational grids that express complicated domain shapes, detailed topography, geographical features, bathymetry and flow structures. High resolution grids require significant memory and computational time. The rapid development of multi-CPU/core parallel architectures with fast networks has dramatically improved the potential for large scale simulations with fast turnaround. In order to take advantage of these parallel computational platforms, it is critical that the computations be scalable. As we increase 
the number of cores, we must consider both the time of the computation and the time required for managing and processing the necessary output files.

The ADCIRC model has been extensively used to model hurricane storm surge along the U.S. East and Gulf coasts by the U.S. Army Corps of Engineers and the Federal Emergency Management Agency for the development of flood risk mitigation systems, flood risk evaluation, and surge forecasting. ADCIRC, a community developed model, is a two and three dimensional coastal ocean hydrodynamic model implemented using both continuous Galerkin (CG) and discontinuous Galerkin (DG) finite element solutions [2]-[15]. The application of basin to channel scale domains using unstructured grids with highly localized resolution is ideal for modeling the riverine, tidal, wind wave, wind, and atmospheric pressure driven flows during a hurricane event. The resulting solutions are both accurate and robust on a wide range of scales and flow phenomena [16]-[22]. For these high resolution computations, ADCIRC has been implemented in parallel using domain decomposition and the MPI (Message Passing Interface) communication library.

In this paper, we report on the scalability of CG based explicit and implicit implementations of ADCIRC in two space dimensions when computing tides and storm surge using large high resolution grids. The number of finite element nodes for the cases examined varies between 254,565 and $9,314,706$, with three degrees of freedom computed at every finite element node every 0.5 second to 2 seconds. We measure parallel scalability on different resolution grids, compare temporal and spatial accuracy for both the implicit and explicit solutions, and evaluate the costs of outputting the 
very large requisite result files. Our simulations were performed on up to 16,384 cores on the Texas Advanced Computing Center (TACC) Ranger machine at the University of Texas at Austin [23]. We analyzed the parallel scalability by instrumenting the code with timers. For the hurricane simulations, results using different time and space resolution were compared and accuracy was evaluated. Since managing output can be extremely costly for the grids examined, we evaluated this aspect as well. It was necessary to designate specialized writer cores which are dedicated to writing output. In order to handle the latency of the disk storage system, we implemented these writer cores in sequential batches which can simultaneously write different types of output files at various solution times.

\section{HURRICANE SIMULATION MODELS}

\section{$2.1 \quad$ Governing Equations}

The two dimensional implementation of the ADCIRC code is based on the vertically integrated shallow water equations [20]. The continuity and momentum equations can be written as follows:

$$
\begin{gathered}
\frac{\partial \zeta}{\partial t}+\nabla \cdot(\mathbf{u} H)=0 \quad \text { on } \Omega^{f}, \\
\frac{\partial \mathbf{u}}{\partial t}+\mathbf{u} \cdot \nabla \mathbf{u}+\tau_{b f} \mathbf{u}+f_{c} \mathbf{k} \times \mathbf{u} \\
+g \nabla \zeta-\nabla \cdot\left[\nu\left\{\nabla \mathbf{u}+(\nabla \mathbf{u})^{T}\right\}\right]=\mathbf{f} \quad \text { on } \Omega^{f},
\end{gathered}
$$


where $\zeta$ is the water surface elevation relative to the geoid, $H \equiv \zeta+$ $h_{b}$ is the total water column depth where $h_{b}$ is the bathymetric depth, and $\mathbf{u}$ represents the depth averaged velocity. The additional external body forces are represented by $\mathbf{f}$, which includes surface stress from wind, variable atmospheric pressure, and tidal potential forcing. $\tau_{b f}, f_{c}, \mathbf{k}, g$ and $\nu$ represent the bottom friction term, the Coriolis parameter, the local vertical vector, the gravitational constant and the lateral eddy viscosity coefficient, respectively.

\subsection{ADCIRC formulation}

The CG implementation of the ADCIRC model uses the generalized wave continuity equation (GWCE) reformulation of the shallow water equations, obtained by combining the continuity equation and the momentum equations as follows:

$$
\begin{gathered}
\frac{\partial^{2} \zeta}{\partial t^{2}}+\tau_{0} \frac{\partial \zeta}{\partial t}+\nabla \cdot\left(\mathbf{J}-g h_{b} \nabla \zeta\right)-\mathbf{u} H \cdot \nabla \tau_{0}=0, \\
\mathbf{J}=\tau_{0} \mathbf{u} H-(\mathbf{u} H) \cdot \nabla \mathbf{u}-\tau_{b f} \mathbf{u} H \\
-f_{c} \mathbf{k} \times \mathbf{u} H-\frac{g}{2} \nabla \zeta^{2}+\mathbf{u} \frac{\partial \zeta}{\partial t}+\nabla \cdot\left[\nu H\left\{\nabla \mathbf{u}+(\nabla \mathbf{u})^{T}\right\}\right]+\mathbf{f} H,
\end{gathered}
$$

where $\tau_{0}$, the so-called GWCE weighting parameter, optimizes numerical accuracy [2], [8]. This so called GWCE equation is solved together with the momentum equation (2).

For the temporal discretization of equation (3), time derivative terms are 
treated as follows:

$$
\begin{gathered}
\frac{\partial^{2} \zeta}{\partial t^{2}}=\frac{\zeta^{n+1}-2 \zeta^{n}+\zeta^{n-1}}{\Delta t^{2}} \\
\frac{\partial \zeta}{\partial t}=\frac{\zeta^{n+1}-\zeta^{n-1}}{2 \Delta t}
\end{gathered}
$$

where $n$ denotes the time level and $\Delta t$ is the time step size. The variable $\zeta$ is weighted over three time levels as follows:

$$
\begin{gathered}
\zeta=\alpha_{1} \zeta^{n+1}+\alpha_{2} \zeta^{n}+\alpha_{3} \zeta^{n-1}, \\
\alpha_{1}+\alpha_{2}+\alpha_{3}=1,
\end{gathered}
$$

where $\alpha_{i}$ is a parameter to control acurracy and stability. The variables $\mathbf{J}$, $\mathbf{u}, \mathbf{f}$ are treated explicitly as known terms in this time step level [8].

The temporal discretization of momentum equation (2) is explicit except for the Coriolis term. The time derivative term and velocity in the bottom friction term and Coriolis force term are treated as:

$$
\begin{gathered}
\frac{\partial \mathbf{u}}{\partial t}=\frac{\mathbf{u}^{n+1}-\mathbf{u}^{n}}{\Delta t}, \\
\mathbf{u}=\frac{1}{2}\left(\mathbf{u}^{n+1}+\mathbf{u}^{n}\right) .
\end{gathered}
$$

The standard Galerkin finite element method is applied for the spatial discretization of equations (3) and (2). The linear $C_{0}$ continuous element 
is used for both $\zeta$ and $\mathbf{u}$.

After the discretization for time and space above, we obtain the following set of linear equations:

$$
\begin{aligned}
& \left(\frac{1}{\Delta t^{2}} \boldsymbol{M}+\frac{1}{2 \Delta t} \boldsymbol{M}_{\bar{\tau}_{0}}+\alpha_{1} \boldsymbol{D}_{\bar{h}_{b}}\right) \Delta \mathbf{Z}^{n+1} \\
& \quad=\left(\frac{1}{\Delta t^{2}} \boldsymbol{M}-\frac{1}{2 \Delta t} \boldsymbol{M}_{\bar{\tau}_{0}}-\left(\alpha_{1}+\alpha_{2}\right) \boldsymbol{D}_{\bar{h}_{b}}\right) \Delta \mathbf{Z}^{n} \\
& \quad+\boldsymbol{D}_{\bar{h}_{b}} Z^{n-1}+\mathcal{Z}^{n} \\
& \left(\frac{1}{\Delta t}+\frac{\tau_{b f}}{2}\right) \boldsymbol{M}_{L} \mathbf{U}^{n+1}+\frac{f_{c}}{2} \boldsymbol{M}_{L}\left(\mathbf{k} \times \mathbf{U}^{n+1}\right) \\
& \quad=\left(\frac{1}{\Delta t}-\frac{\tau_{b f}}{2}\right) \boldsymbol{M}_{L} \mathbf{U}^{n}-\frac{f_{c}}{2} \boldsymbol{M}_{L}\left(\mathbf{k} \times \mathbf{U}^{n}\right)+\mathcal{U}^{n},
\end{aligned}
$$

where $\mathbf{Z}, \mathbf{U}$ represent the nodal vector(s) of $\zeta, \mathbf{u} . \Delta(:)^{n+1}$ indicates the difference on this time step level. $\mathcal{Z}^{n}, \mathcal{U}^{n}$ are known nodal vectors on this time level. Normally, we solve the GWCE implicitly and we set the temporal control parameters to $\alpha_{1}=\alpha_{2}=\alpha_{3}=\frac{1}{3}$. Equation (11) is solved using a conjugate gradient iterative solver with diagonal scaling system based on Compressed Row Storage (CRS) [24]. The discrete momentum equations (12) are solved explicitly and apply a lumped mass matrix, $\boldsymbol{M}_{L}$, and are therefore matrix free. Details are discussed in [8]. We designate this scheme as the implicit solution in this paper. On the other hand, if we set the temporal control parameters in equation (11) to $\alpha_{1}=\alpha_{3}=0$, $\alpha_{2}=1$, we can solve the discrete GWCE system explicitly using the lumped mass matrix in the GWCE. This results in an entirely matrix free solution for both $\zeta$ and $\mathbf{u}$, reducing both computational time and memory. We designate this as the explicit solution. 


\subsection{Code parallelization}

The ADCIRC parallel computation is based on a domain decomposition paradigm. The computational domain is divided into sub-domains with one overlapping layer of finite elements between adjacent sub-domains as shown as Figure 1. Each sub-domain is allocated to an individual core. METIS [25], which is based on graph theory, is used as the domain decomposer. For the parallel computation, two types of inter-subdomain and inter-core communication are required. Communication is necessary between the neighboring sub-domains to complete the correct assembly of the subdomain nodal matrices and vectors and to ensure that all subdomain vectors match the global vectors. The data on the inside node of the overlapping layer is sent to the neighboring sub-domain, and the data on the external edge of the layer is received from the neighboring sub-domain. In addition, communication among all sub-domains is required to calculate a summation over the entire global domain. This communication step is necessary for the dot product in the iterative solver in order to evaluate the residual norm of the solution. There are two dot products in each conjugate gradient iteration and generally 10-15 iterations are required to reach convergence. Therefore, the implicit solution requires 20 to 30 global communication procedures in one time step. On the other hand, this global communication procedure does not appear in the explicit solution.

For these communications, MPI (Message Passing Interface) is used. Specifically for communication between adjacent subdomains, MPI_ISEND and MPI_IRECV are used in both the implicit and explicit solution, while MPI_ALLREDUCE is used for the necessary global communication for the 
implicit solution.

\subsection{Application of sequential batches of dedicated writer cores}

Our standard implementation of managing output simply consists of a globalization process of output from all cores to core number $0(\mathrm{C} 0)$ which then writes the information to the disk storage system. On machines such as the CRAY XT3 (Sapphire, [26]) and the CRAY XT4 (Jade, [27]), the procedure works well, largely due to the high level output caching and output data management by the operating system. On TACC's Lonestar and Ranger machines, the standard output procedure takes considerable time, often more than doubling the wall clock time. The problem is that the output file data is as large as approximately 100 Bytes per finite element node per output time interval, which amounts to approximately 1.0 GBytes per output time interval for a grid with 9,314,706 nodes when writing surface water elevations, water velocities, atmospheric pressure, and wind speeds. A typical output time interval is 15 to 30 minutes. The time required to write this data to the disk array is large, sometimes substantially exceeding the actual computational time required between output time intervals. The problem is aggravated when using large numbers of cores.

Figure 2 shows the time schedule for the computation and writing procedure using the standard output procedure for two output files. All cores are compute cores, and the results of each sub-domain are gathered to compute core number $0(\mathrm{C} 0)$. Core $\mathrm{C} 0$ then outputs the result to the disk storage system. The other compute cores can not proceed to the next time step 
until core $\mathrm{C} 0$ finishes writing the results.

We therefore investigated the use of dedicated writer cores to reduce the total wall clock time for the simulation which includes the computation time in addition to the required file writing time. The specialized writer cores do not handle any portion of the actual finite element computation and have no assigned sub-domain. These cores devote themselves to collecting the output data from all the compute cores, globalizing the necessary files and then writing these files to the disk storage system.

The time schedule of the dedicated writer core algorithm is shown in Figure 3 . The compute cores can move to the next step immediately after they have sent their results to the specialized writer cores. We can have one writer core handling all output files, or one writer core per output file, effectively distributing the output load. The writer cores output the results to the disk array while the compute cores proceed with the finite element computation. If the output time is longer than the simulation time, the compute cores do have to wait until the writer cores finish writing because the compute cores cannot send their data. For this situation, we simply have to use a larger total number of writer cores than the number of output files. Figure 4 shows a case with four writer cores for two output files. Defining the sequential batches of writer cores allows each batch to complete the writing process over several writing output time intervals. When a batch of writer cores completes the writing procedure, they become available to handle another set of output files. The compute cores can send the result data to the next batch of writer cores without stopping.

We note that for all implementations of the dedicated writer cores, the time required to send data to the appropriate writer cores interrupts the 
computation. However the computation is not slowed down by the time needed to do the actual writing to the disk storage system, which is substantially longer than the data collection and globalization time.

\subsection{Flow of the computation}

The flow chart of the computation is shown in Figure 5. The computation during a time step can be detailed as follows:

1) The left hand side matrix and right hand side vector of equation (11) are calculated and solved using a conjugate gradient method in the implicit solution. For the explicit solution, the left hand side matrix uses a lumped mass matrix (i.e. terms only on the diagonal) and is therefore matrix free.

2) If the domain has a lateral moving boundary, a wet/dry judgment is required to update the active wet finite elements. For the implicit solution, a global Allreduce is required to determine if elements have changed from wet to dry or dry to wet so that this information can be used to reconstruct and solve the global matrix. No such call is necessary for the explicit solution.

3) The right hand side vector of equation (12) is calculated and the system is solved using a lumped mass matrix, resulting in a matrix free solution for velocity.

4) The computed results are output to the disk storage system at required output intervals.

5) The time step loop 1) 4) is repeated until the final time step. 


\subsection{Computer system specifications}

We used the Sun Blade cluster based supercomputer Ranger at TACC at the University of Texas at Austin [23]. Ranger has 3,936 compute nodes, each node has four quadcore AMD Opteron 8356 processors for a total of 62,976 cores. All compute nodes are interconnected with a $1 \mathrm{~GB} / \mathrm{s}$ InfiniBand network. The specifications of each compute node are shown in Table 1. CentOS 4.8(x86_64) is used as the operating system, and we used PGI Fortran 4.2.1 and MVAPICH 1.0 as the Fortran compiler and MPI Library.

\section{Performance Benchmarks}

\subsection{Benchmark problems}

We used two different base grids in order to evaluate the performance of both the implicit and explicit implementation of the CG GWCE based ADCIRC solution. The first is the SL15 grid which was developed to evaluate tidal, riverine, wind wave and hurricane induced storm surge response in coastal Louisiana and Mississippi [21][22]. The model applies a finite element grid with elements that range between $30 \mathrm{~m}$ and $24 \mathrm{~km}$. The model considers finite elements changing from wet to dry or dry to wet states simulating a lateral wetting and drying moving boundary. In addition, the model incorporates flow overtopping weirs handled as internal barrier boundaries. The second grid is the EC2001 model which was developed to study tides in the western North Atlantic, Gulf of Mexico and Caribbean Sea and applies finite elements ranging between $150 \mathrm{~m}$ and $24 \mathrm{~km}$ [19]. The EC2001 model does not include the wetting and drying process or internal 
barrier boundaries.

The SL15x01 grid, shown in Figure 6, applies large elements in deep ocean water, an intermediate level of resolution on continental shelves and a high level of resolution in coastal Louisiana and Mississippi, the region of specific interest. Figure 6 also shows a sample domain decomposition, the bold lines designating the borders of the 1,024 sub-domains. The areal extent of the sub-domains varies dramatically and is inversely proportional to grid size. The EC2001 grid, shown in Figure 7, shows less variability in grid size and therefore more uniformity in the areal coverage of the sub-domains. We refined these two base grids into higher resolution implementations by dividing the base grid elements into 4 and 16 elements as illustrated in Figure 8. We use x01, x04 and x16 as suffixes of the grid names to differentiate the grids with the original and indicate the level of increased resolution. The grids and the total number of nodes and elements are summarized in Table 2.

In order to study CG ADCIRC's parallel performance and scalability, tidal simulations were performed on the SL15x01, x04 and the EC2001x01, x04, x16 grids. Hurricane storm surge simulations were carried out for the SL15x01, x04 grids to quantify temporal and spatial accuracy and to compare the results of the implicit and explicit solutions. The latter simulations were also used to quantify the performance of the standard output and dedicated writer core output options.

\subsection{Parallel performance}

In order to measure the parallel performance of the CG implementation of ADCIRC, tidal flow simulations were carried out on Ranger. Output file 
writing was suppressed for these simulations. We measured the wall clock time using 16 to 16,384 cores for 6 hours of real time simulation. A time step, $\Delta t=1 \mathrm{~s}$ was used for the SL15x01 grid, and $0.5 \mathrm{~s}$ was used for the SL15x04 grid in order to maintain stability as well as accuracy. Table 3 summarizes runtime information for the implicit and the explicit solutions on the SL15x01 and SL15x04 grids. The wall clock times presented in this table and the related figures are normalized to 1day of simulation using a 1s time step. Figure 9 shows a comparison of the wall clock times of the implicit and the explicit solutions for both grids as a function of the total number of cores. We can see that wall clock time is inversely proportional to the number of cores over a wide range of cores. We note that the slopes of these curves often exceed the ideal slope of minus one over a range of cores. This is largely related to caching efficiencies which are most consistently noted in the increased rate of scaling between 256 and 512 cores for both the implicit and explicit solutions of the SL15x01 grid, and between 1024 and 2048 cores for the implicit and explicit solutions of the SL15x04 grid. Ultimately the ideal scaling on all the grids is lost although this occurs later for the larger SL15x04 grid and also occurs later and more slowly for the explicit solution as compared to the implicit solution. Furthermore, we note that the explicit solution is about twice as fast as the implicit solution on a per time step basis from a small number of cores until the curves start to tail off, at which point the difference becomes larger.

Figure 10 highlights these features by plotting wall clock times against the number of finite element nodes per core. Again the wall clock time is normalized to 1day of simulation using a 1s time step. We note that now the performance of the SL15x01 and SL15x04 grids is very similar for 
the implicit solution as it is for the explicit solution. Overall performance appears to be controlled by the number of finite element nodes per core for each of these solutions. We note approximately ideal scaling for all solutions with the better than ideal scaling between 4,500 and 9,000 finite element nodes per core, corresponding to the aforementioned caching efficiencies. Degradation in ideal scaling occurs quite rapidly for the implicit solution at about 2,200 finite element nodes per core and much more slowly for the explicit solution at about 1,100 finite element nodes per core. Thus both solutions exhibit weak scaling, i.e. the implicit solutions for the two grids overlay each other as do the explicit solutions. Furthermore, strong scaling characteristics for individual solutions are sustained until about 1,100 to 2,200 finite element nodes per core although the degradation in ideal scaling is much slower for the explicit solution.

Figure 11 shows the parallel speedup obtained when doubling the number of cores in intervals between 16 and 16,384 plotted as a function of the total number of finite element nodes per core. Parallel speedup achieved when doubling the number of cores is defined as:

$$
\text { Parallel Speedup } N_{2 c}=\frac{\text { Time }_{N_{c}}}{\text { Time }_{N_{2 c}}},
$$

where $N_{c}$ is the total number of cores and $N_{2 c}$ is twice the number of cores. The ideal parallel speedup is equal to 2.0. Between 300,000 and 10,000 finite element nodes per core, the ideal speedup is achieved and often episodically improved upon, due to improvements in caching and memory access. At 4500 finite element nodes per core, reflecting the scaling between 9000 and 4500 finite element nodes per core, we note a consistent boost beyond 
ideal speedup for all solutions, reflecting significant improvements in cache utilization that occur in this range of finite element nodes per core. For fewer than 2,200 finite element nodes per core, a degradation in efficiency occurs at various rates.

A detailed analysis of the core utilization for the SL15x04 grid is presented for the implicit and explicit solution in Figure 12 and 13 respectively. The total wall clock time, the wall clock time of the solution of the continuity equation, the momentum equations, the wetting and drying judgment, the local communication (Send and Receive), and the global communication (Allreduce) are shown for the implicit solution as a function of the total number of cores. Identical information is shown for the explicit solution with the exception of the global communication time which is not required for the explicit solution. We note that for the implicit solution both the total solution time, continuity equation solution time and the wetting/drying judgment exhibit degradation in scalability with large numbers of cores while the momentum equation solution sees only a modest reduction in scalability at 8,192 cores. More importantly while the local communication sees some limited growth at 4,096 cores, the global communication time sees steady increases over the entire range of cores. In fact, the global communication time becomes a dominating contributor to the total time as the total number of cores grows. We note that there may be some effect of the timers on the recorded simulation times. The explicit solution in Figure 13 shows perfect scaling for both the continuity equation and the momentum equation solution time up to 8,192 cores and then a degradation in the scaling efficiency. While there is no global communication, there is a slow down in the rate of decrease in the local 
communication at about 4,096 cores.

The global communication (Allreduce) time increases with the number of cores and this influence appears in the time for the continuity equation solution as well as for the wetting and drying judgment procedure in the implicit method. On the other hand, the explicit solution, which does not include the Allreduce communication, retains good scalability. However, even the explicit solution exhibits an eventual degradation in scalability. This degradation was also seen in the explicit solution of the momentum equation in our implicit solution procedure. This reduction in scalability is caused by a reduction in scaling efficiency of the local communications time as well as the increase in the relative number of overlapping subdomain finite element nodes, as measured by the ratio of the total number of finite element nodes summed over all sub-domains to the total number of finite element nodes in the original grid. Figure 14 shows this ratio and indicates the importance of the number of additional overlapping finite element nodes as the number of finite element nodes per core decreases below 1,000 to 2,000 .

We now compare tidal simulations run with the EC2001 grids for one day using time steps: $\Delta t=2.0$ s for the EC2001x01 grid; 1.0s for the EC2001x04 grid; and 0.5s for the EC2001x16 grid. Table 4 summarizes runtime information for the implicit and the explicit solutions on the EC2001x01, x04 and x16 grids. The wall clock time shown in this table and the related figures are also normalized to 1day of simulation using a 1s time step. We compare wall clock times as a function of the number of cores in Figure 15. We note the linear or better than linear scaling caused by the cache effect. The most pronounced manifestation of the cache effect occurs between 64 
and 128 cores for the EC2001x01 grid; between 256 and 512 cores for the EC2001x04 grid; and between 1,024 and 2,048 cores for the EC2001x16 grid. Ultimately all solutions lose strong scaling. However weak scaling is evident in that the more finite element nodes a grid has, the longer it maintains its scaling. Furthermore, the explicit solutions maintain their scaling much longer than the implicit solutions, and the performance improvement as compared to the implicit solution becomes larger as the solutions' scalablity start to tail off. The implicit solution loses ideal scaling before 512 cores on the EC2001x01 grid, before 2,048 cores on the x04 grid, and before 8,192 cores on the x16 grid. After that, the wall clock times on all grids increase to similar times, which is the effect of the increasing global Allreduce communication times, the effect of local communications, as well as the increasing numbers of overlapping finite element nodes. The explicit solution loses scalability before 4,096 cores on the EC2001x01 grid, before 8,192 cores on $\mathrm{x} 04$ grid, and before 16,384 cores on x16 grid. The saturation of wall clock time occurs at higher number of cores due to the increase in the total number of finite element nodes originating from the overlapping sub-domains as well as local communication time.

Figure 16 shows that all EC2001 grids behave similarly for the implicit and explicit solutions when wall clock time is measured using the number of finite element nodes per core. We again note that the explicit solution is about twice as fast as the implicit solution, that all solutions have a well defined cache boost, and that the explicit solution scales much longer than the implicit solution. Figure 17 plots the parallel speed up as a function of the number of finite element nodes per core. Both Figure 16 and Figure 17 suggest that for the EC2001 computations, the cache boost occurs at 
about 2000 finite element nodes per core, reflecting that a boost occurs somewhere between 4000 and 2000 finite element nodes per core. The optimal cache boost for the SL15 grids occured at approximately double that number of finite element nodes per core, although the discrete doubling increments make the exact boost points difficult to determine. In addition, the scalability is sustained longer for the EC2001 computations than for the SL15 computations. The reduction in scalability occurs below 1000 finite element nodes per core and is caused by a slow down in the scaling of the local communication time as well as the increase in the relative number of overlapping sub-domain finite element nodes. Figure 18 shows this ratio and indicates the importance of the number of additional overlapping finite element nodes as the number of finite element nodes per core decreases below 1,000. We also note that the EC2001 computations are about twice faster than the SL15 computations on a wall clock time per finite element node per core basis. The shift in the optimal cache boost, the difference in simulation times, and the delayed reduction in scalability reflect the fact that the SL15 computations include wetting and drying, have much smaller finite elements, and include internal barrier boundaries. This implies stronger nonlinear terms, resetting matrices, and more iterations for the Conjugate Gradient matrix solver (for the implicit solution). In addition, the wetting and drying algorithm and the internal barrier logic require substantial computing time.

\subsection{Comparison of results}

In order to evaluate the time stepping accuracy, spatial accuracy, as well as the difference between the implicit and explicit solution, we examine 
the differences between the various solutions for a simulation of the storm surge that developed during Hurricane Katrina [21][22].

First, in order to check the stability and accuracy of the time discretizations, we show a comparison of the water surface elevations near the peak of the storm surge using different time step sizes for the SL15x01 grid. Figure 19 shows the distribution of water surface elevation and forcing wind velocity vectors as the storm is developing the peak storm surge on the SL15x01 grid for the implicit solution. Figure 20 shows the difference in water surface elevation using an implicit solution with $1 \mathrm{~s}$ and $0.5 \mathrm{~s}$ time steps, and Figure 21 shows the difference in elevation using an explicit solution with $1 \mathrm{~s}$ and $0.5 \mathrm{~s}$ time steps. In these figures, there are no significant differences in the numerical solutions and we can conclude that the selected time steps are stable and accurate.

Next, we show a comparison of water surface elevations on grids with different spatial resolutions. Figure 22 shows the difference in water elevation between the SL15x01 grid and SL15x04 grid for the implicit solution, and Figure 23 shows this difference for the explicit solution. We used a 0.5 s time step for both the SL15x01 grid and the SL15x04 grid. We note that in the regions marked I in Figure 22,23 there are differences in the limited blue areas where the SL15x04 grid solution is larger than the SL15x01 grid solution. These areas have very shallow flow depths and have recently been subject to the wetting and drying moving boundary. The wetting and drying process converges as element size reduces and is therefore better represented by the finer SL15x04 grid. Thus the SL15x01 grid needs a higher density of elements around these areas. There is also a very limited underprediction in the SL15x01 grid in region II, where overtopping weir 
flow, computed using internal barrier boundary conditions, is greater in the finer SL15x04 grid. Again the finer grid solution improves the solution. Region III is at the interface of wind blowing water away from a levee and gradient driven high water pushing its way towards the same levee. This also represents a wet and dry interface which is again better represented by the higher resolution grid.

Finally, we note that the spatial errors between the lower and higher resolution SL15x01 and SL15x04 grids are essentially identical for implicit and explicit solutions. A comparison between the implicit and explicit solutions reflects this and we can not see significant differences between these solutions with the exception of small regions near wet and dry interfaces, as shown in Figure 24.

\subsection{Model Validation}

The SL15 model has been extensively validated against available tide, river flow - stage, and hurricane Katrina and Rita high water mark (HWM) and hydrograph data [21][22]. The hurricane hindcasts were forced with tides on the Atlantic open ocean boundary, tidal potential functions that are derived from solar and lunar gravitational forces, riverine flows, atmospheric pressure, wind fields, and wind wave forces. The wind wave forcing is computed through two way couplings to nested wave models and is especially important in wave transformation zones. The SL15x01 grid applied in this paper is an incremental refinement of the earlier SL15 model and has been revalidated by simulating Hurricane Katrina using optimized data assimilated wind fields [21]. Figures 25 and 26 compare the computed high water during Hurricane Katrina to data collected by the U.S. Army Corps of 
Engineers as well as by URS Corporation for the Federal Emergency Management Agency (FEMA) [28] [29]. The collected HWM's are distributed throughout coastal and inland southeastern Louisiana and Mississippi. The figures indicate that there is essentially no bias (with best fit slopes of 0.99 and 1.02) and that there is a close fit between the computed and measured data (with correlation coefficients squared equal to 0.92 and 0.94 respectively).

\subsection{File writing performance}

In order to measure the performance of the dedicated writer cores, the Hurricane Katrina storm surge simulation [21][22] was benchmarked with the various writing options described in section 2.4. The wall clock times were measured for a three hour real time simulation on the SL15x04 grid with four output files (water surface elevation, the two components of the velocity vector, the two components of the wind forcing vector, and atmospheric pressure). Each output file was written to every fifteen minutes of real time simulation (every 1,800 time steps) for all finite element nodes on the global grid. Table 5 shows the wall clock times of the implicit and explicit solution using 1,024 cores and 8,192 cores with no output, standard output, with 1 dedicated writer core, 2 dedicated writer cores, 4 dedicated writer cores, and 8 dedicated writer cores organized in 2 sequential batches. In this table, the number inside of the parentheses shows the communication time used in writing the four output files, specifically the time required for the compute cores to send the result data to the writer cores.

Figure 27 shows the wall clock times for the implicit solution. The standard output option, which uses one compute core as the data output col- 
lector and writer, almost doubles the wall clock time due to disk array latency. In this figure, the significant improvement achieved using the dedicated writer core algorithm is seen compared with the standard output option. We note that the actual required writing time of the implicit solution with 1,024 cores using either 1,2,4, and 8 writer cores is less than the computation time for any given output time interval. For the 8,192 core case the actual required writing time to the four output files exceeds the computation wall clock time for the output interval as the parallel scaling has dramatically reduced (by a factor of 3.86) the computation wall clock time. Therefore more dedicated writer cores are needed; in this case, two suffices.

The explicit solution case shown in Figure 28 needs even more dedicated writer cores for effective file output. For the 1,024 core case, the total run time almost triples when the standard output option is used. We note that the required total writing time is the same as for the implicit 1,024 core case (approximately 1,085 seconds), but that the relative proportion of the total run spent writing is larger. The use of one writer core is therefore insufficient since the computation time between output intervals is smaller than the total required writing time. Therefore at least two writer cores are required. As can be seen in Figure 28 and Table 5, distributing the writing process on two or more dedicated writer cores dramatically improves overall performance. The explicit solution with 8,192 total cores exhibits the most dramatic slow down when the standard output option is used, by more than a factor of 12. It is clear that the scalable performance of this simulation is destroyed by the file writing process. The 8,192 core explicit computation time is in fact 7.3 times faster than the 1,024 core computation time while 
the total required file output time is still about 1050 seconds for both simulations. As noted in Figure 28 and Table 5, we now require 8 writer cores organized in two sequential batches, which are simultaneously writing 2 sets of output files, to achieve improvements in the total wall clock time.

We note that the best total time with the 2 sequential batches of 4 writer cores still exceeds the total simulation time without any writing. This is due to the fact that the output data send time from the compute cores to the writer cores (which varies between about 60 seconds and 112 seconds) is asynchronous and is performed by the compute cores which can not be computing while performing this operation as was illustrated in Figure 4. Furthermore we note from Table 5 that the data send time does not scale, either with the number of compute cores, or the number of writer cores. The ideal time then is the no output time plus the data send time. This is almost achieved, as seen in Table 5, although there is a discrepancy between the best total time and the ideal time which varies between 5 seconds (for the 8,192 core, explicit case) and 97 seconds (for the 8,192 core, implicit case). This is likely related to data send time syncronization which can be related to the variable data load that must be transmitted and the congestion of network traffic.

\section{CONCLUSIONS}

The scalability of the continuous Galerkin implementation of the ADCIRC unstructured grid shallow water equation model was measured for various forcing function scenarios and on different resolution grids. Both implicit and explicit implementations were examined. The implicit code shows 
strong scaling on the TACC Ranger machine with up to about 2,000 finite element nodes per core. The code also exhibits weak scaling with scalability being sustained as long as the problem size is increased and at least 2,000 finite element nodes per core are used. The explicit solution is at least twice as fast as the implicit solution on a per finite element node basis and exhibits better and longer scalability since this solution does not require the global communication that limits the scalability of the implicit solution. Ultimately as the number of finite element nodes per core decrease, all solutions exhibit a deterioration in scalability due to local communication times as well as a significant increase in the number of additional overlapping finite element nodes at the subdomain interfaces. Finally, both the implicit and explicit solutions experience a substantial cache boost when between 2,000 and 4,000 finite element nodes per core are specified.

It is apparent that the required large scalar and vector output files for water levels, currents, wind speed and atmospheric pressure can destroy scalability and dramatically increase wall clock times. A dedicated writer core algorithm was proposed for effective output file writing in order to improve the total performance of the simulation with large and/or frequent output file access. The writer core algorithm can use sequential batches of writer cores so that sets of output files at sequential times can be written synchronously. The dedicated writer cores contributed to the dramatic improvement in simulation times effectively almost regaining simulation times with no output with the exception of the required global data transfer time between the compute cores and the dedicated writer cores which does not scale.

It appears that the explicit solutions are an excellent choice for improving 
wall clock times and sustaining scalability for large grids when a large number of processors are available. The explicit solution may also be an attractive option when performing simulations on a system with relatively slow communications links. Comparisons between the implicit and explicit solutions indicate that the solutions are almost identical when time steps are selected that are sufficiently small to ensure accuracy and stability.

It is also clear that wall clock times can be achieved that allow the CG ADCIRC model to be used for forecasting hurricane storm surge. A 9,314,706 finite element node grid with 1s time steps can complete a day of real time simulation in between 200 and 400 wall clock seconds for the computation plus approximately 400s of output communication time using 16,384 cores and the sequential batch dedicated writer core algorithm to output water surface elevations, velocities, winds, and pressure every 30 min of real time. This suggests that a very high resolution simulation can be achieved in less than 20 min of wall clock time per day of real time simulation, well within forecasting time window requirements. Larger finite element grids can also be used due to the weak scaling characteristics and the continued development of output file management which can help further reduce the file output communication times.

\section{Acknowledgements}

This work was supported by awards from the National Science Foundation (OCI-0749015, OCI-0746232, DMS-0620696, DMS-0620697 and DMS0620791) and the Office of Naval Research (N00014-06-1-0285). Computational resources were provided in part by awards from TACC and the TeraGrid project (TGDMS080016N). 


\section{References}

[1] Resio, D.T. and Westerink, J.J. (2008). Hurricanes and the Physics of Surges. Physics Today, 61, 9, 33-38.

[2] Kolar, R.L., Westerink, J.J., Cantekin, M.E. and Blain, C.A. (1994). Aspects of nonlinear simulations using shallow-water models based on the wave continuity equation. Computers and Fluids, 23, 523-528.

[3] Luettich, R.A., Hu, S. and Westerink, J.J. (1994). Development of the direct stress solution technique for three dimensional hydrodynamic models using finite elements. International Journal for Numerical Methods in Fluids, 19, 295-319.

[4] Grenier, R.R., Luettich, R.A. and Westerink, J.J. (1995). A comparison of the nonlinear frictional characteristics of two-dimensional and three-dimensional models of a shallow tidal embayment. Journal of Geophysical Research, 100, C7, 13719-13735.

[5] Kolar, R.L., Gray, W.G. and Westerink, J.J. (1996). Boundary conditions in shallow water models - an alternative implementation for finite element codes. International Journal for Numerical Methods in Fluids, 22, 603-618.

[6] Atkinson, J.H., Westerink, J.J. and Hervouet, J.M. (2004). Similarities between the quasi-bubble and the generalized wave continuity equation solutions to the shallow water equations. International Journal for Numerical Methods in Fluids, 45, 689-714.

[7] Atkinson, J.H., Westerink, J.J., Luettich, R.A. (2004). Twodimensional dispersion analyses of finite element approximations to the 
shallow water equations. International Journal for Numerical Methods in Fluids, 45, 715-749.

[8] Luettich, R.A. and Westerink, J.J. (2004). Formulation and Numerical Implementation of the 2D/3D ADCIRC Finite Element Model Version 44.XX; http://adcirc.org/adcirc_theory_2004_12_08.pdf

[9] Dawson, C., Westerink, J.J., Feyen, J.C. and Pothina, D. (2006). Continuous, discontinuous and coupled discontinuous-continuous Galerkin finite element methods for the shallow water equations. International Journal for Numerical Methods in Fluids, 52, 63-88.

[10] Kubatko, E.J., Westerink, J.J. and Dawson, C. (2006). hp discontinuous Galerkin methods for advection dominated problems in shallow water flow. Computer Methods in Applied Mechanics and Engineering, 196, 437-451.

[11] Kubatko, E.J., Westerink, J.J. and Dawson, C. (2007). Semi-discrete discontinuous Galerkin methods and stage exceeding order strong stability preserving Runge-Kutta time discretizations. Journal of Computational Physics, 222, 832-848.

[12] Kubatko, E.J., Dawson, C. and Westerink, J.J. (2008). Time step restrictions for Runge-Kutta discontinuous Galerkin methods on triangular grids. Journal Computational Physics, 227, 9697-9710.

[13] Kubatko, E.J., Bunya, S., Dawson, C., Westerink, J.J. and Mirabito C. (2009). A performance comparison of continuous and discontinuous finite element shallow water models. Journal of Scientific Computing. 40, 315-339. 
[14] Bunya, S., Kubatko, E.J., Westerink, J.J. and Dawson, C. (2009). A wetting and drying treatment for the Runge-Kutta discontinuous Galerkin solution to the shallow water equations. Computer Methods in Applied Mechanics and Engineering, 198, 1548-1562.

[15] Kubatko, E.J., Bunya, S., Dawson, C. and Westerink, J.J. (2009). Dynamic p-adaptive Runge-Kutta discontinuous Galerkin methods for the shallow water equations. Computer Methods in Applied Mechanics and Engineering, 198, 1766-1774.

[16] Westerink, J.J., Luettich, R.A. and J.C. Muccino, J.C. (1994). Modeling tides in the western North Atlantic using unstructured graded grids. Tellus, 46A, 178-199.

[17] Blain, C.A., Westerink, J.J. and Luettich, R.A. (1994). The influence of domain size on the response characteristics of a hurricane storm surge model. Journal of Geophysical Research, 99, C9, 18467-18479.

[18] Blain, C.A., Westerink, J.J. and Luettich, R.A. (1998). Grid convergence studies for the prediction of hurricane storm surge. International Journal for Numerical Methods in Fluids, 26, 369-401.

[19] Mukai, A., Westerink, J.J., Luettich, R.A. and Mark, D. (2002). Eastcoast 2001: A tidal constituent database for the western North Atlantic, Gulf of Mexico, and Caribbean Sea. Tec. Rep. ERDC/CHL TR-02-24, U.S.Army Corps of Engineers, 25.

[20] Westerink, J.J., Luettich, R.A., Feyen, J.C., Atkinson, J.H., Dawson, C., Roberts, H.J., Powell, M.D., Dunion, J.P., Kubatko, E.J., Pourtaheri, H. (2008). A basin to channel scale unstructured grid hurricane 
storm surge model applied to Southern Louisiana. Monthly Weather Review, 136, 3, 833-864.

[21] Bunya, S., Dietrich, J.C., Westerink, J.J., Ebersole, B.A., Smith, J.M., Atkinson, J.H., Jensen, R., Resio, D.T., Luettich, R.A., Dawson, C., Cardone, V.J., Cox, A.T., Powell, M.D., Westerink, H.J., Roberts, H.J. (2009). A high resolution coupled riverine flow tide, wind, wind wave and storm surge model for Southern Louisiana and Mississippi: part I - model development and validation. Monthly Weather Review, DOI: $10.1175 / 2009$ MWR2907.1, in press.

[22] Dietrich, J.C., Bunya, S., Westerink, J.J., Ebersole, B.A., Smith, J.M., Atkinson, J.H., Jensen, R., Resio, D.T., Luettich, R.A., Dawson, C., Cardone, V.J., Cox, A.T., Powell, M.D., Westerink, H.J., Roberts, H.J. (2009). A high resolution coupled riverine flow tide, wind, wind wave and storm surge model for Southern Louisiana and Mississippi: part II - synoptic description and analyses of hurricane Katrina and Rita. Monthly Weather Review, DOI: 10.1175/2009MWR2906.1, in press.

[23] http://www.tacc.utexas.edu/

[24] Kincaid, D.R., Respess, J.R., Young, D.M. and Grimes, R.G. (1982). ITPACK 2C: A Fortran package for solving large sparse linear systems by adaptive accelerated iterative methods, ACM Transactions on Mathematical Software, 8, No.3. 
[25] Karypis, G. and Kumar, V. (1998). Multilevel k-way partitioning scheme for irregular graphs. Journal of Parallel and Distributed Computing. 48, No.1. 96-129.

[26] http://www.erdc.hpc.mil/systemNews/Cray_XT3/home

[27] http://www.erdc.hpc.mil/systemNews/Cray_XT4/home

[28] URS, (2006). Final coastal and riverine high-water marks collection for Hurricane Katrina in Louisiana. FEMA-1603-DR-LA, Task Orders 412 and 419, Federal Emergency Management Agency, Washington DC, 76pp.

[29] Ebersole, B.A., Westerink, J.J., Resio, D.T., and Dean, R.G. (2007). Performance Evaluation of the New Orleans and Southeast Louisiana Hurricane Protection System, Volume IV - The Storm. Final Report of the Interagency Performance Evaluation Task Force, U.S. Army Corps of Engineers, Washington, D.C., 263 pp. 
Table 1: Specifications of TACC Ranger compute nodes

\begin{tabular}{lll}
\hline \hline Sun Blade x6420 & CPU & 4 Quadcore AMD Opteron 8356 \\
\hline & Memory & $16 \times 2 \mathrm{~GB}$ DDR2-667 ECC-reg \\
\hline \hline AMD Opteron 8356 & Frequency & $2.3 \mathrm{GHz}$ \\
\hline & Architecture & AMD K10 (Barcelona) \\
\hline & L1-Cache & $64+64 \mathrm{~KB}$ per core \\
\hline & L2-Cache & $512 \mathrm{~KB}$ per core \\
\hline & L3-Cache & $2048 \mathrm{~KB}$ on die shared \\
\hline
\end{tabular}

Table 2: Finite element grids

\begin{tabular}{crrrcc}
\hline \hline Grid name & $N_{\text {node }}$ & $N_{\text {elem }}$ & $h_{e}$ & Wet/Dry & Internal Barrier \\
\hline \hline SL15x01 & $2,351,832$ & $4,611,048$ & $30 \mathrm{~m}$ & yes & yes \\
\hline SL15x04 & $9,314,706$ & $18,444,184$ & $15 \mathrm{~m}$ & yes & yes \\
\hline \hline EC2001x01 & 254,565 & 492,179 & $200 \mathrm{~m}$ & no & no \\
\hline EC2001x04 & $1,001,418$ & $1,968,716$ & $100 \mathrm{~m}$ & no & no \\
\hline EC2001x16 & $3,971,661$ & $7,874,864$ & $50 \mathrm{~m}$ & no & no \\
\hline
\end{tabular}

${ }^{*} N_{\text {node }}$ : total number of nodes, $N_{\text {elem }}$ : total number of elements, $h_{e}:$ minimum element size 
Table 3: Runtime information of one day simulation on the SL15x01 and SL15x04 grids normalized to use $1 s$ time steps.

\begin{tabular}{|c|c|c|c|c|c|}
\hline \multicolumn{6}{|c|}{ SL15x01 } \\
\hline \multirow{2}{*}{$N_{\text {core }}$} & \multirow{2}{*}{$N_{n / c}$} & \multicolumn{2}{|c|}{ Implicit scheme } & \multicolumn{2}{|c|}{ Explicit scheme } \\
\hline & & Wallclock time $[\mathrm{s}]$ & Speed up & Wallclock time $[\mathrm{s}]$ & Speed up \\
\hline 16 & 148,068 & 120731.61 & - & 60678.24 & - \\
\hline 32 & 74,343 & 59850.76 & 2.017 & 29700.53 & 2.043 \\
\hline 64 & 37,429 & 25590.49 & 2.339 & 12236.35 & 2.427 \\
\hline 128 & 18,895 & 11684.77 & 2.190 & 6144.74 & 1.991 \\
\hline 256 & 9,581 & 5745.79 & 2.034 & 2853.01 & 2.154 \\
\hline 512 & 4,885 & 2166.24 & 2.652 & 1230.39 & 2.319 \\
\hline 1,024 & 2,508 & 1230.39 & 1.761 & 637.32 & 1.931 \\
\hline 2,048 & 1,301 & 834.30 & 1.475 & 364.64 & 1.748 \\
\hline 4,096 & 684 & 682.78 & 1.222 & 266.04 & 1.371 \\
\hline 8,192 & 365 & 689.45 & 0.990 & 203.73 & 1.306 \\
\hline 16,384 & 199 & 626.08 & 1.101 & 196.63 & 1.036 \\
\hline \multicolumn{6}{|c|}{ SL15x04 } \\
\hline \multirow{2}{*}{$N_{\text {core }}$} & \multirow{2}{*}{$N_{n / c}$} & \multicolumn{2}{|c|}{ Implicit scheme } & \multicolumn{2}{|c|}{ Explicit scheme } \\
\hline & & Wallclock time $[\mathrm{s}]$ & Speed up & Wallclock time $[\mathrm{s}]$ & Speed up \\
\hline 16 & 584,097 & 566901.85 & - & 193604.45 & - \\
\hline 32 & 292,642 & 254135.47 & 2.231 & 96533.11 & 2.006 \\
\hline 64 & 146,767 & 120788.46 & 2.104 & 48395.24 & 1.995 \\
\hline 128 & 73,722 & 53113.71 & 2.274 & 24779.52 & 1.953 \\
\hline 256 & 37,104 & 26528.93 & 2.002 & 12480.74 & 1.985 \\
\hline 512 & 18,727 & 12753.84 & 2.080 & 6244.20 & 1.999 \\
\hline 1,024 & 9,491 & 5863.19 & 2.175 & 2988.20 & 2.090 \\
\hline 2,048 & 4,834 & 2699.35 & 2.172 & 1341.23 & 2.228 \\
\hline 4,096 & 2,479 & 1652.44 & 1.634 & 774.00 & 1.733 \\
\hline 8,192 & 1,283 & 879.92 & 1.878 & 492.13 & 1.573 \\
\hline 16,384 & 672 & 736.81 & 1.194 & 385.21 & 1.278 \\
\hline
\end{tabular}

$* N_{\text {core }}$ : total number of cores, $N_{n / c}$ : total number of nodes per core. Speed up refers to the speed up achieved when doubling the number of cores and is defined in Eq.(13). 
Table 4: Runtime information of one day simulation on the EC2001x01, $\mathrm{x} 04$ and $\mathrm{x} 16$ grids normalized to use $1 s$ time steps.

\begin{tabular}{|c|c|c|c|c|c|}
\hline \multicolumn{6}{|c|}{ EC2001x01 } \\
\hline \multirow{2}{*}{$N_{\text {core }}$} & \multirow{2}{*}{$N_{n / c}$} & \multicolumn{2}{|c|}{ Implicit scheme } & \multicolumn{2}{|c|}{ Explicit scheme } \\
\hline & & Wallclock time $[\mathrm{s}]$ & Speed up & Wallclock time $[\mathrm{s}]$ & Speed up \\
\hline 16 & 16,113 & 6350.71 & - & 2794.17 & - \\
\hline 32 & 8,142 & 2992.93 & 2.122 & 1427.00 & 1.958 \\
\hline 64 & 4,127 & 1518.40 & 1.971 & 662.35 & 2.154 \\
\hline 128 & 2,107 & 625.45 & 2.428 & 251.22 & 2.637 \\
\hline 256 & 1,088 & 344.49 & 1.816 & 127.25 & 1.974 \\
\hline 512 & 567 & 245.86 & 1.401 & 72.33 & 1.759 \\
\hline 1,024 & 302 & 234.96 & 1.046 & 49.91 & 1.449 \\
\hline 2,048 & 165 & 230.44 & 1.020 & 31.66 & 1.577 \\
\hline 4,096 & 92 & 270.93 & 0.851 & 27.71 & 1.142 \\
\hline 8,192 & 54 & 274.43 & 0.987 & 37.25 & 0.744 \\
\hline 16,384 & 34 & 363.68 & 0.755 & 32.23 & 1.156 \\
\hline \multicolumn{6}{|c|}{ EC2001x04 } \\
\hline \multirow{2}{*}{$N_{\text {core }}$} & \multirow{2}{*}{$N_{n / c}$} & \multicolumn{2}{|c|}{ Implicit scheme } & \multicolumn{2}{|c|}{ Explicit scheme } \\
\hline & & Wallclock time $[\mathrm{s}]$ & Speed up & Wallclock time $[\mathrm{s}]$ & Speed up \\
\hline 16 & 63,041 & 26626.87 & - & 11275.86 & - \\
\hline 32 & 31,643 & 12951.64 & 2.056 & 5583.80 & 2.019 \\
\hline 64 & 15,934 & 6344.41 & 2.041 & 2770.21 & 2.016 \\
\hline 128 & 8,059 & 3081.70 & 2.059 & 1416.87 & 1.955 \\
\hline 256 & 4,094 & 1635.11 & 1.885 & 770.18 & 1.840 \\
\hline 512 & 2,094 & 628.21 & 2.603 & 271.07 & 2.841 \\
\hline 1,024 & 1,083 & 316.92 & 1.982 & 156.66 & 1.730 \\
\hline 2,048 & 566 & 236.33 & 1.341 & 101.73 & 1.540 \\
\hline 4,096 & 302 & 170.90 & 1.383 & 56.73 & 1.793 \\
\hline 8,192 & 165 & 185.71 & 0.920 & 38.03 & 1.491 \\
\hline 16,384 & 93 & 282.04 & 0.658 & 36.82 & 1.033 \\
\hline \multicolumn{6}{|c|}{ EC2001x16 } \\
\hline \multirow{2}{*}{$N_{\text {core }}$} & \multirow{2}{*}{$N_{n / c}$} & \multicolumn{2}{|c|}{ Implicit scheme } & \multicolumn{2}{|c|}{ Explicit scheme } \\
\hline & & Wallclock time $[\mathrm{s}]$ & Speed up & Wallclock time $[\mathrm{s}]$ & Speed up \\
\hline 16 & 249,047 & 134239.24 & - & 45027.21 & - \\
\hline 32 & 124,745 & 58465.68 & 2.296 & 22437.68 & 2.007 \\
\hline 64 & 62,639 & 25229.53 & 2.317 & 11290.12 & 1.987 \\
\hline 128 & 31,486 & 12520.63 & 2.015 & 5683.99 & 1.986 \\
\hline 256 & 15,873 & 6149.89 & 2.036 & 2760.32 & 2.059 \\
\hline 512 & 8,035 & 3125.73 & 1.968 & 1506.56 & 1.832 \\
\hline 1,024 & 4,088 & 1593.64 & 1.961 & 754.09 & 1.998 \\
\hline 2,048 & 2,094 & 637.40 & 2.500 & 282.16 & 2.673 \\
\hline 4,096 & 1,084 & 380.80 & 1.674 & 150.97 & 1.869 \\
\hline 8,192 & 568 & 323.35 & 1.178 & 94.14 & 1.604 \\
\hline 16,384 & 303 & 356.81 & 0.906 & 70.03 & 1.344 \\
\hline
\end{tabular}

$* N_{\text {core }}$ : total number of cores, $N_{n / c}$ : total number of nodes per core. Speed up refers to the speed up achieved when doubling the number of cores and is defined in Eq.(13). 
Table 5: Wall clock times of three hour hurricane simulation with four output files written every 15 min of simulation time and communication (send/receive) time used for writing (in parenthesis).

\begin{tabular}{ccrrrrr|r}
\hline \hline & no output & standard & 1 wcore & 2 wcore & 4 wcore & 8 wcore & ideal \\
\hline \hline Implicit & 1506.27 & 2590.86 & 1583.30 & 1611.09 & 1598.07 & 1612.17 & 1573 \\
1,024 & & & $(63.19)$ & $(63.91)$ & $(66.39)$ & $(66.98)$ & \\
\hline Implicit & \multirow{2}{*}{150.18} & 1467.59 & 977.18 & 549.76 & 504.42 & 576.17 & 481 \\
8,192 & & & $(89.24)$ & $(88.56)$ & $(90.75)$ & $(88.50)$ & \\
\hline Explicit & 679.93 & 1767.81 & 1033.56 & 837.01 & 869.16 & 865.71 & 792 \\
1,024 & & & $(95.98)$ & $(94.38)$ & $(102.88)$ & $(112.17)$ & \\
\hline Explicit & 92.86 & 1134.85 & 974.49 & 536.57 & 356.83 & 186.47 & 181 \\
8,192 & & & $(85.24)$ & $(78.22)$ & $(81.62)$ & $(88.50)$ & \\
\hline \hline
\end{tabular}




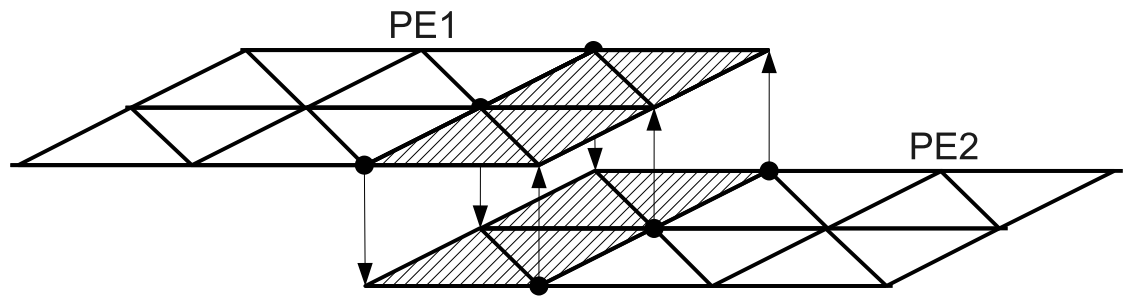

Figure 1: Overlapping adjacent sub-domains with the communication flow of the variables from internal overlapping layer finite element nodes to external overlapping layer finite element nodes.

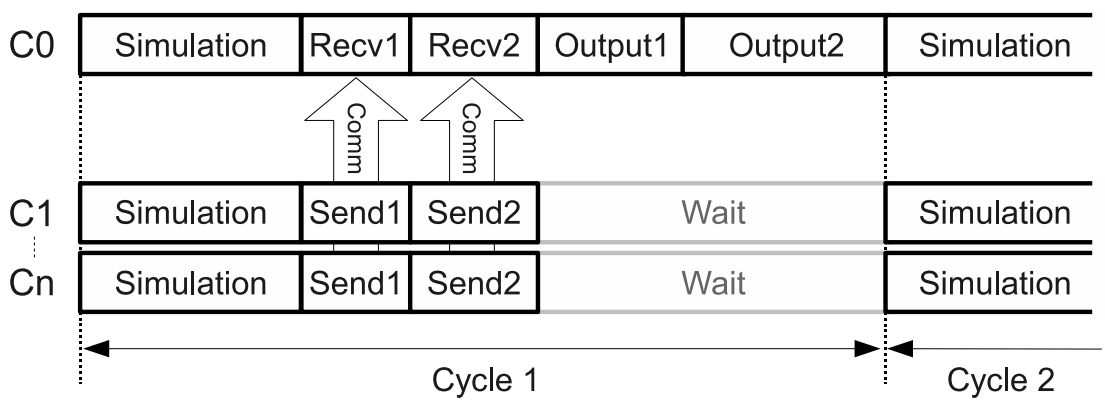

Figure 2: Time schedule of each core for the standard output algorithm.

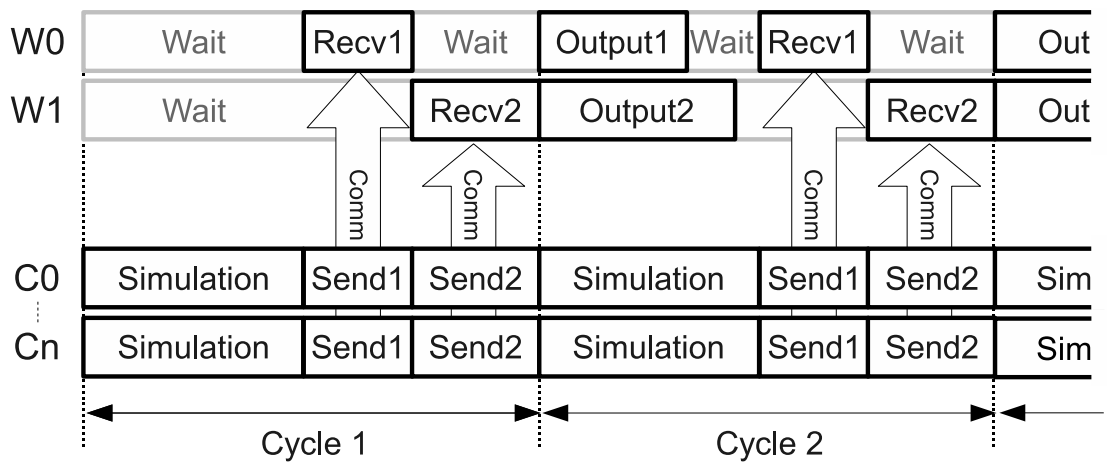

Figure 3: Time schedule of each core for the dedicated writer core algorithm. 


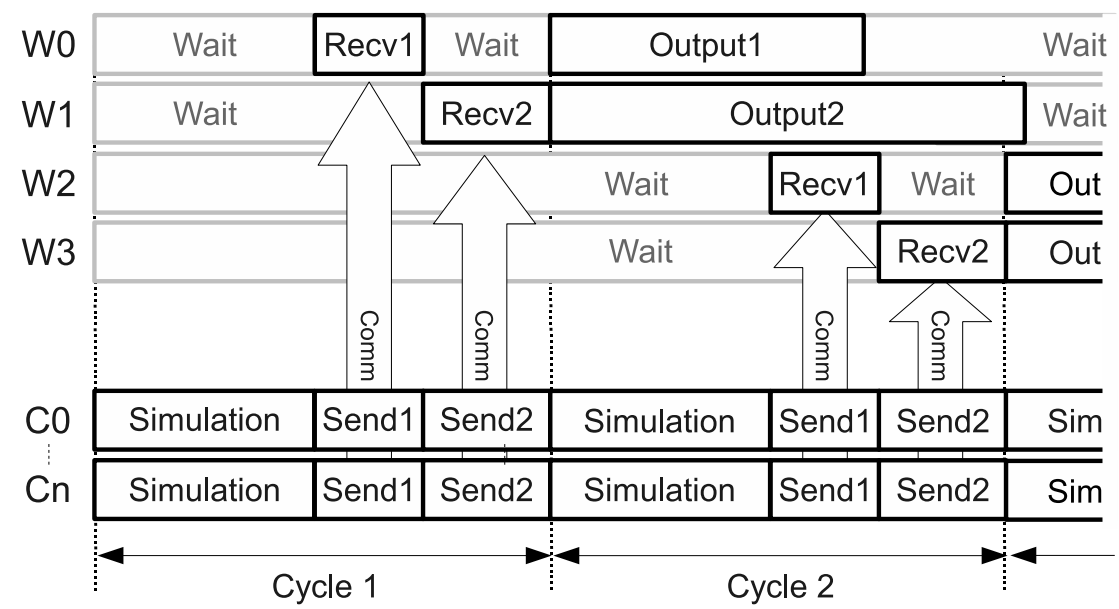

Figure 4: Time schedule of each core for the dedicated writer core algorithm organized in two sequential batches. 


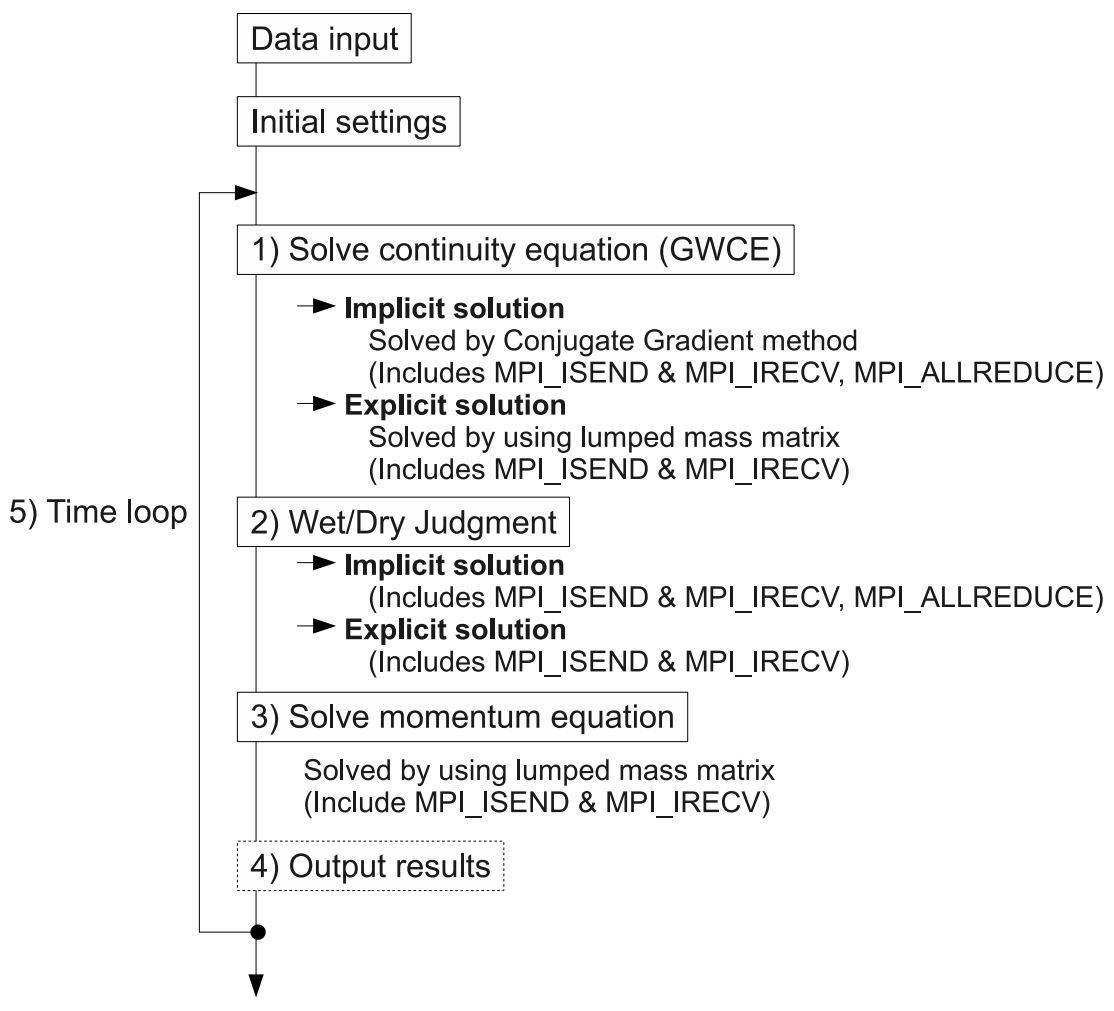

Figure 5: Flow chart of the simulation. 


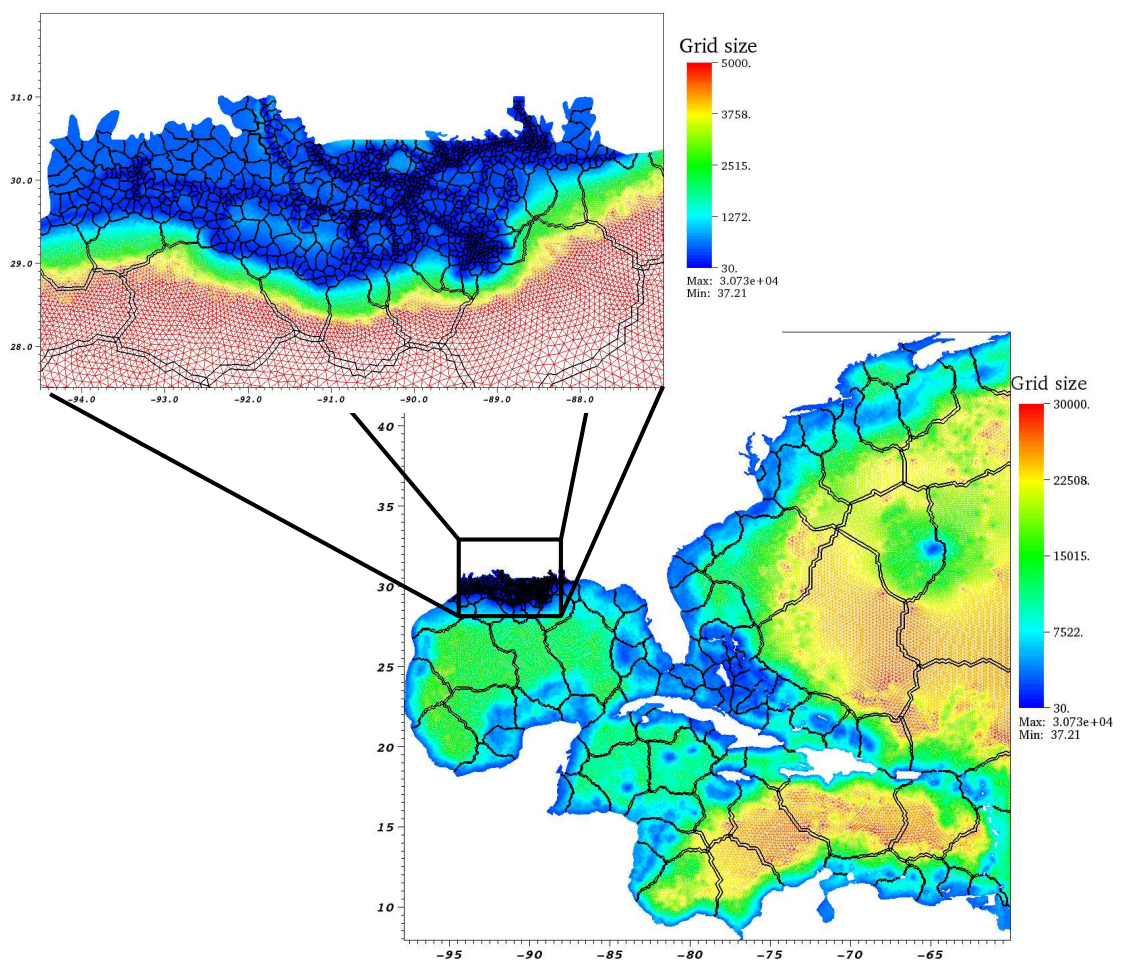

Figure 6: SL15x01 grid colored by finite element size and the borders of 1,024 subdomains. 


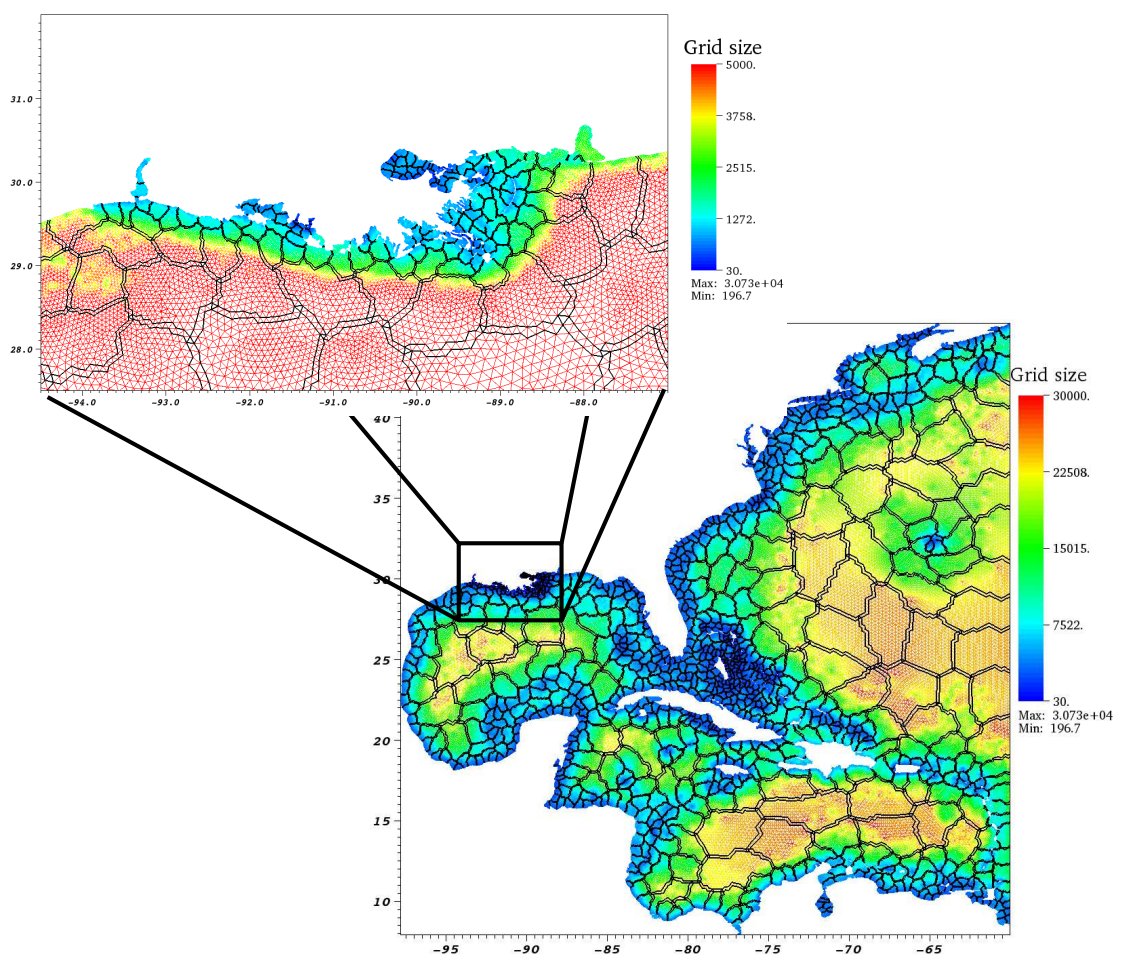

Figure 7: EC2001x01 grid colored by finite element size and the borders of 1,024 subdomains.

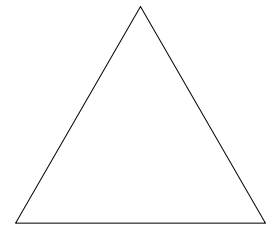

$\mathrm{x} 01$

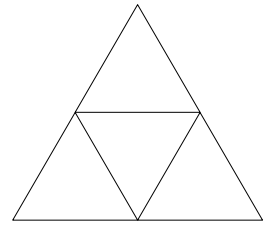

$\mathrm{x} 04$

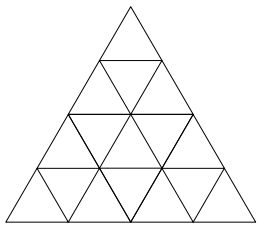

$\mathrm{x} 16$

Figure 8: Refinement of grids. 


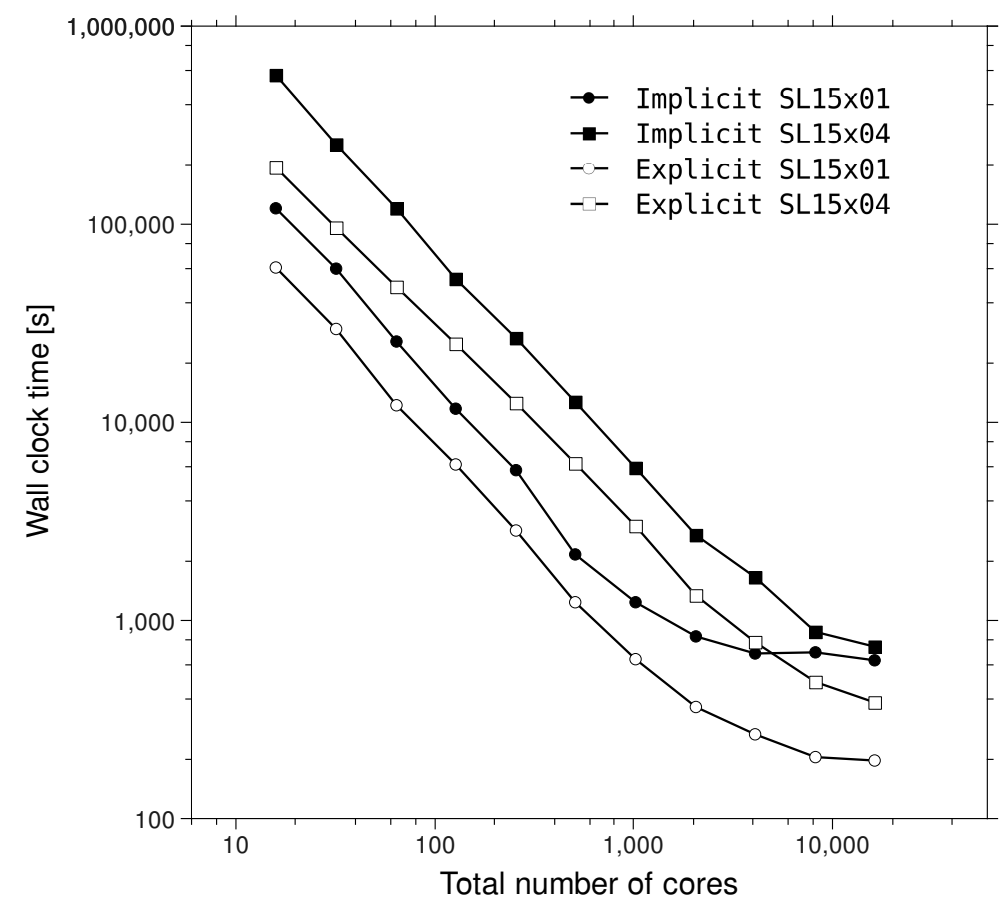

Figure 9: Comparison of the wall clock times (s) of the implicit and the explicit solution on the SL15x01, x04 grids as a function of the total number of cores. Wall clock times are normalized to 1 day of real time using $1 \mathrm{~s}$ time steps. 


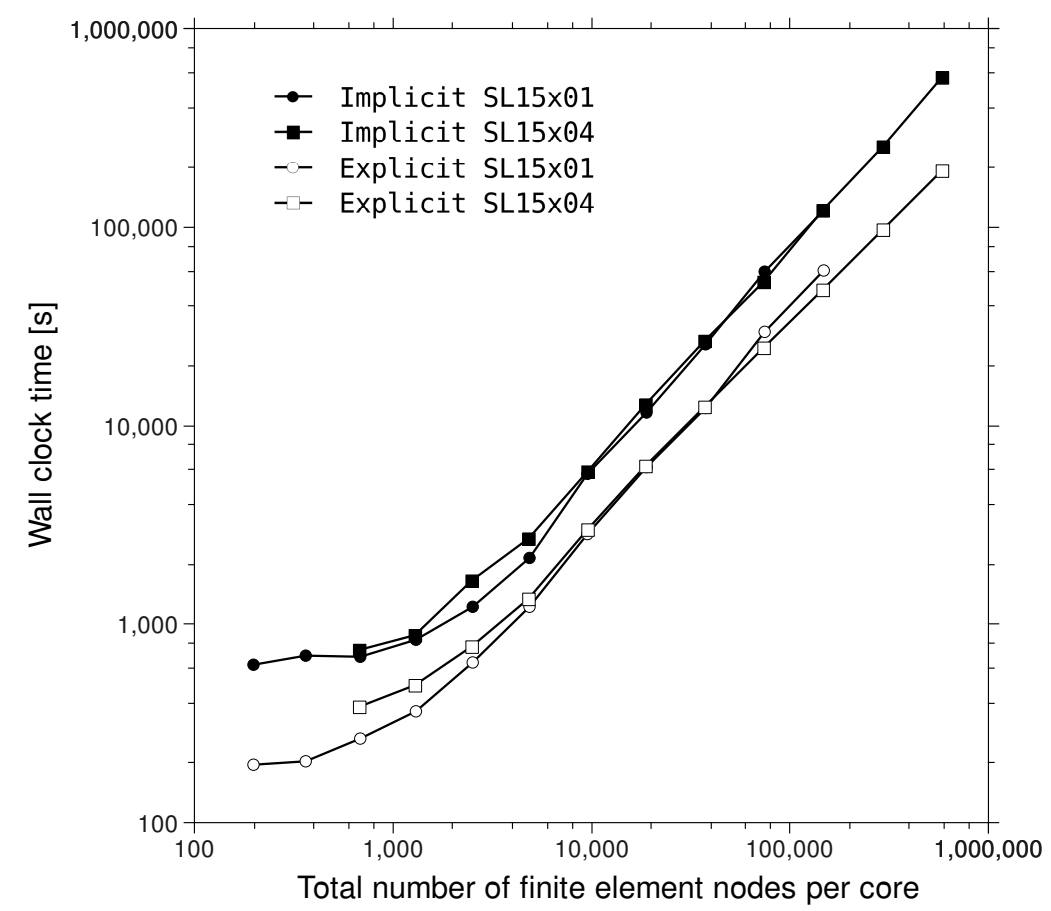

Figure 10: Comparison of the wall clock times of the implicit and the explicit solution on the SL15x01, x04 grids as a function of the total number of finite element nodes per core. Wall clock times are normalized to 1 day of real time using 1 s time steps. 


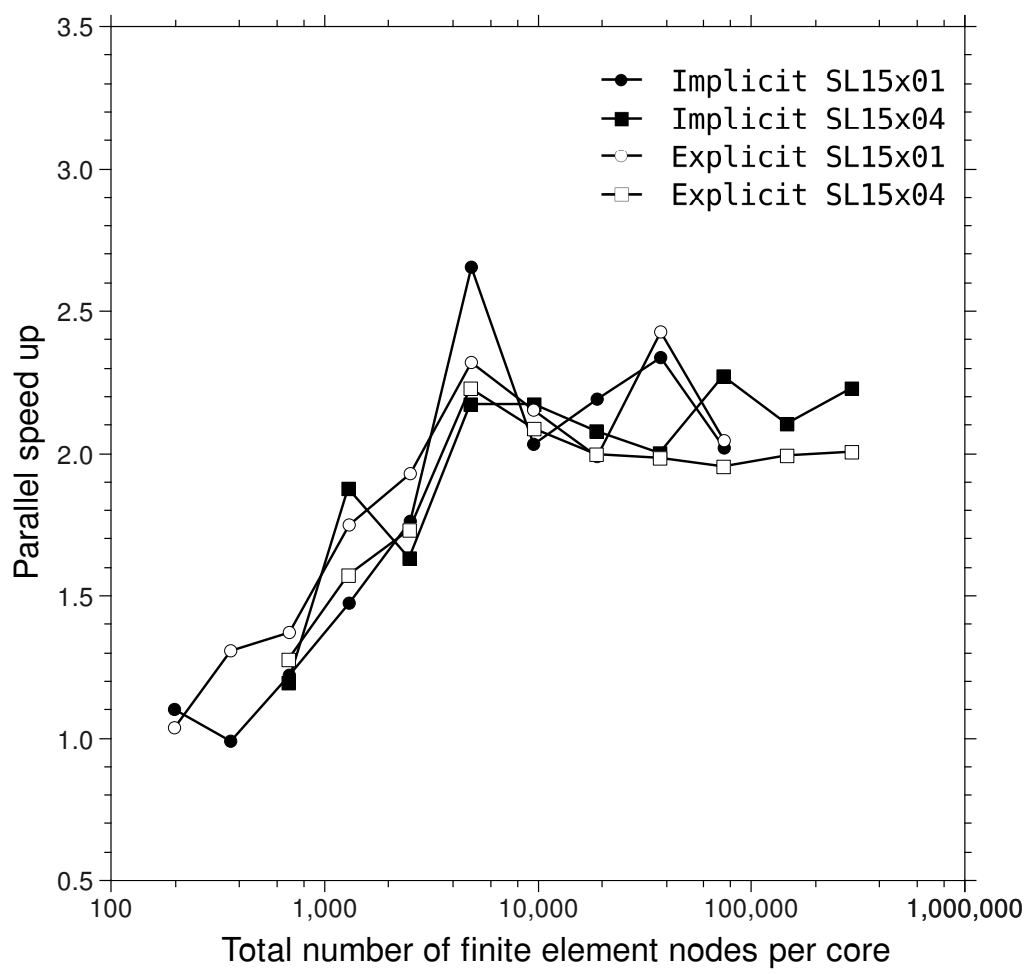

Figure 11: Parallel speed up resulting from core doubling for implicit and explicit solutions of the SL15x01, x04 grids as a function of the total number of finite element nodes per core. 


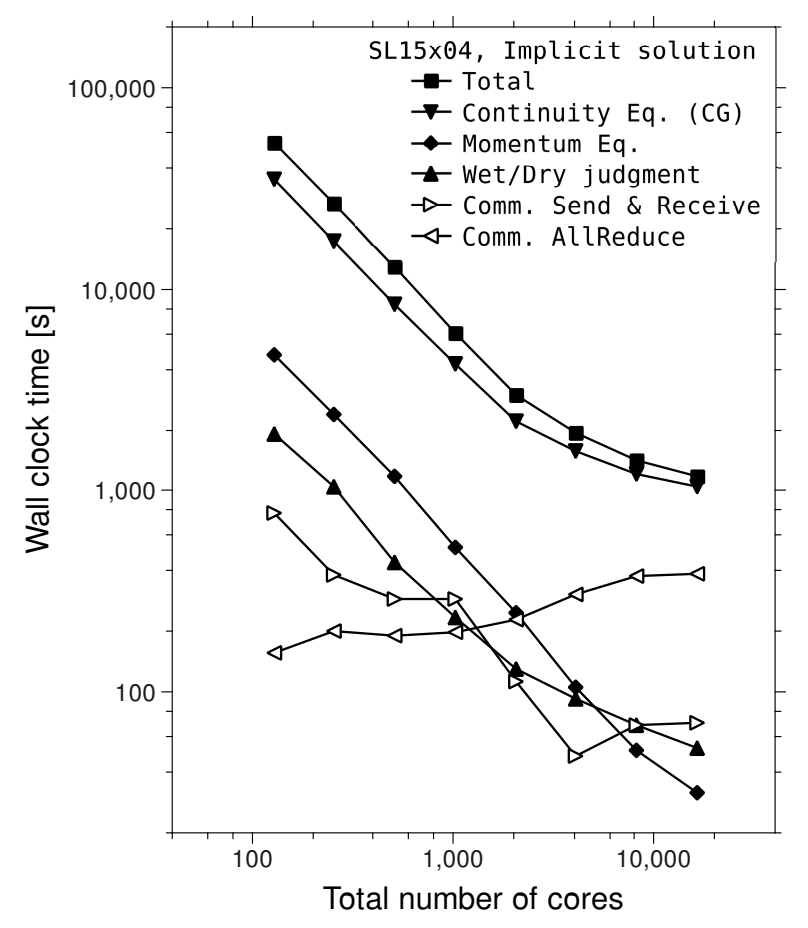

Figure 12: Component computational times of the implicit solution of the SL15x04 grid. 


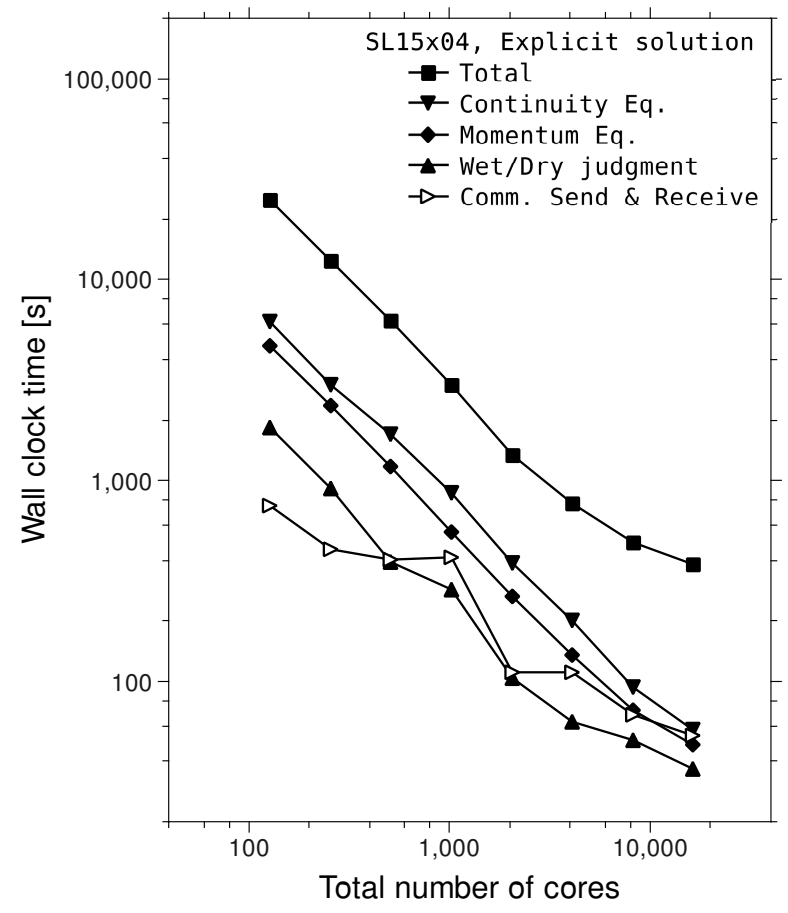

Figure 13: Component computational times of the explicit solution of the SL15x04 grid. 


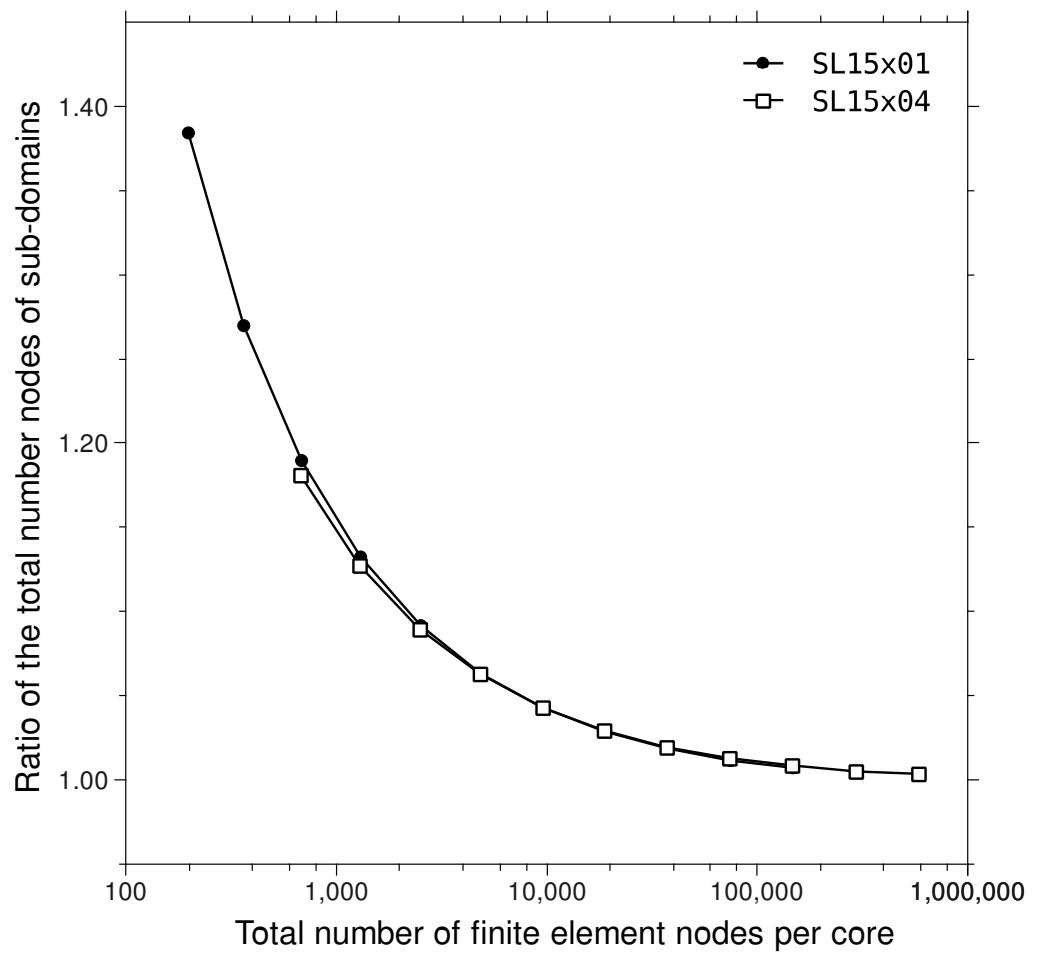

Figure 14: The ratio of the sum of the total number of finite element nodes on all sub-domains to the original number of finite element nodes in the global grid for the SL15 grids as a function of the total number of finite element nodes per core. 


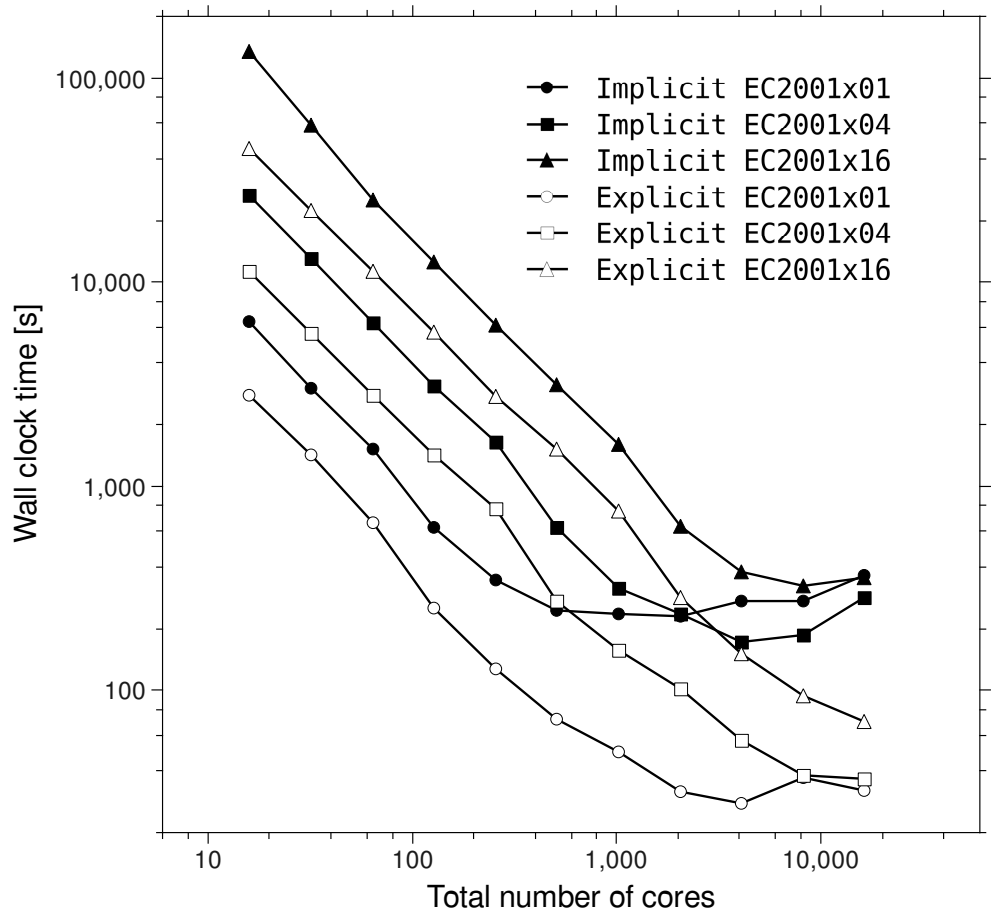

Figure 15: Comparison of the wall clock times (s) of the implicit and the explicit solution of the EC2001x01, x04, x16 grids as a function of the total number of cores. Wall clock times are normalized to 1 day of real time using 1s time steps. 


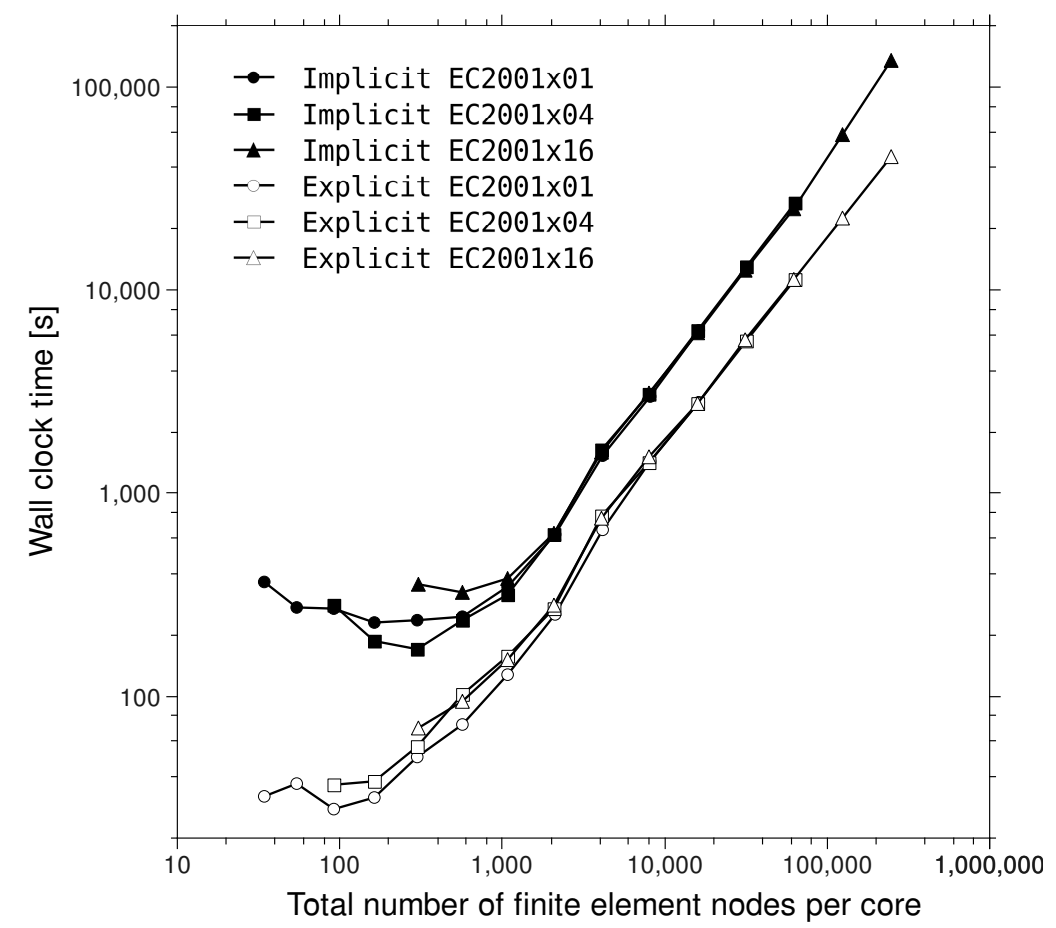

Figure 16: Comparison of the wall clock times of the implicit and the explicit solution on the EC2001x01, x04, x16 grids as a function of the total number of finite element nodes per core. Wall clock times are normalized to 1 day of real time using 1 s time steps. 


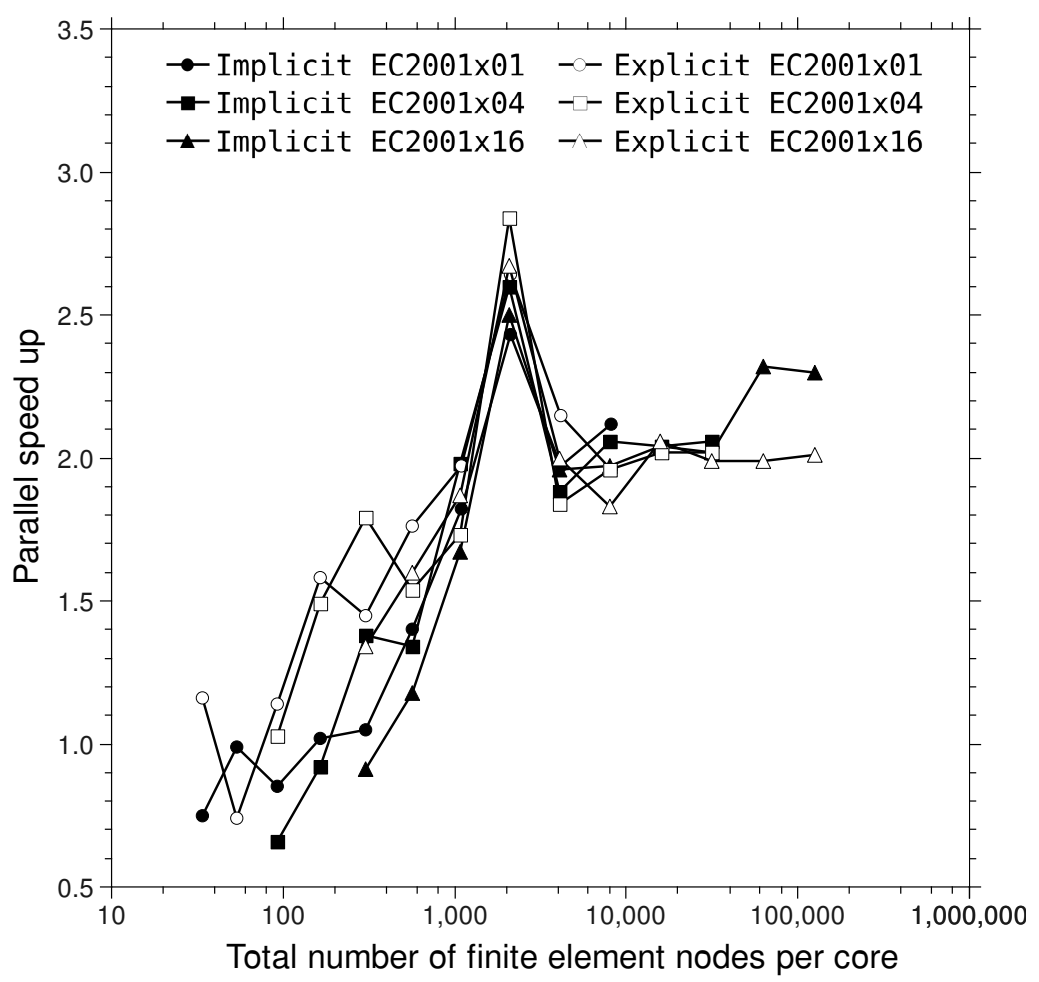

Figure 17: Parallel speed up resulting from core doubling for implicit and explicit solutions of the EC2001x01, x04, x16 grids as a function of the total number of finite element nodes per core. 


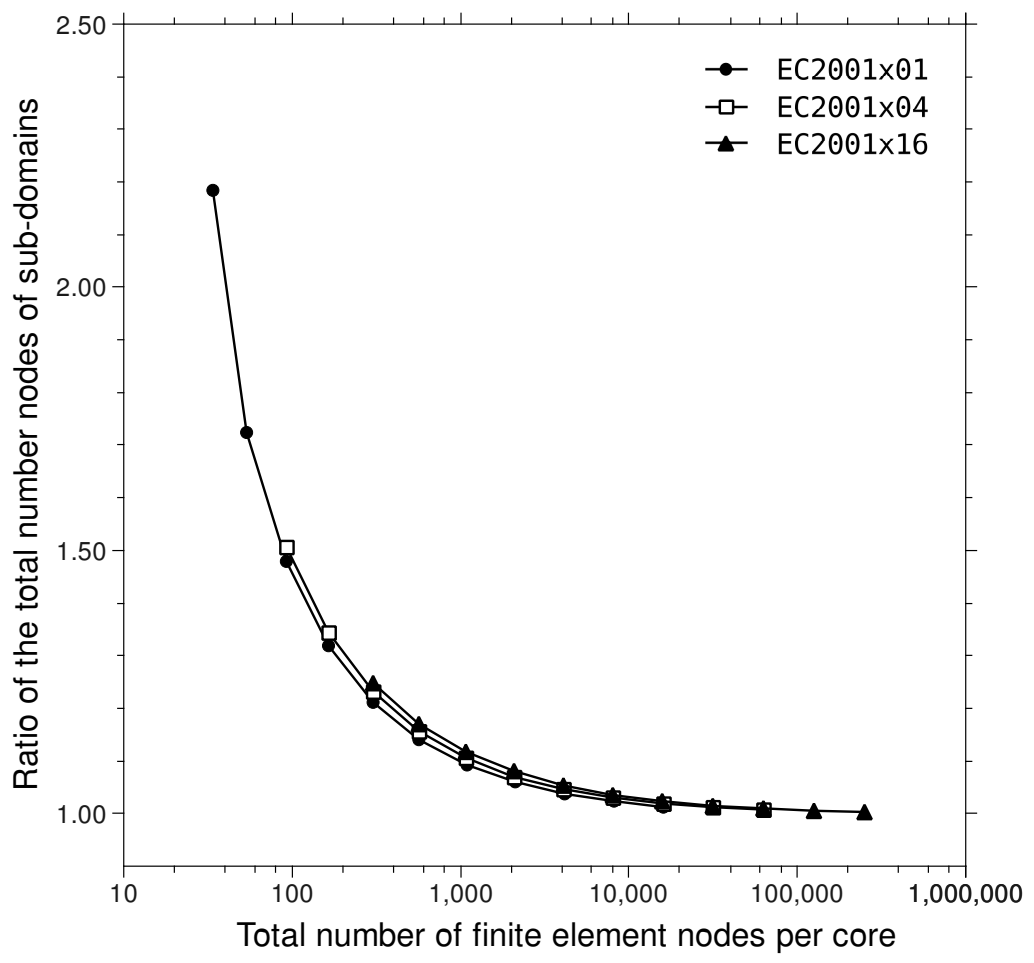

Figure 18: The ratio of the sum of the total number of finite element nodes on all sub-domains to the original number of finite element nodes in the global grid for the EC2001 grids as a function of the total number of finite element nodes per core. 


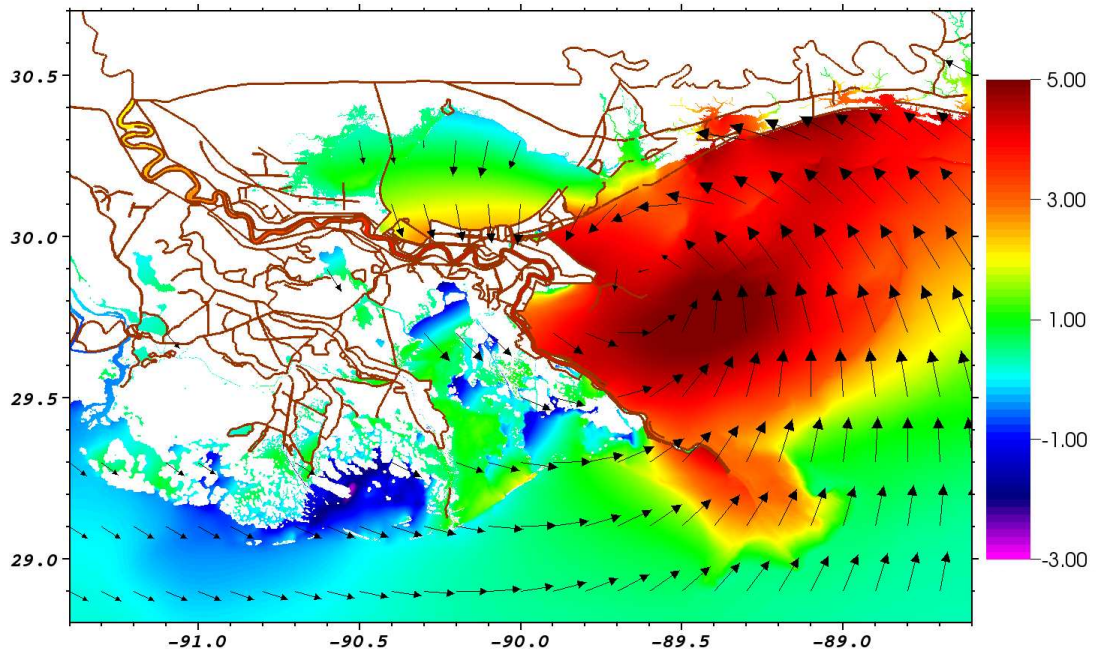

Figure 19: Water surface elevation $(\mathrm{m})$ and wind velocity vectors $(\mathrm{m} / \mathrm{s})$ near the peak of the storm surge.

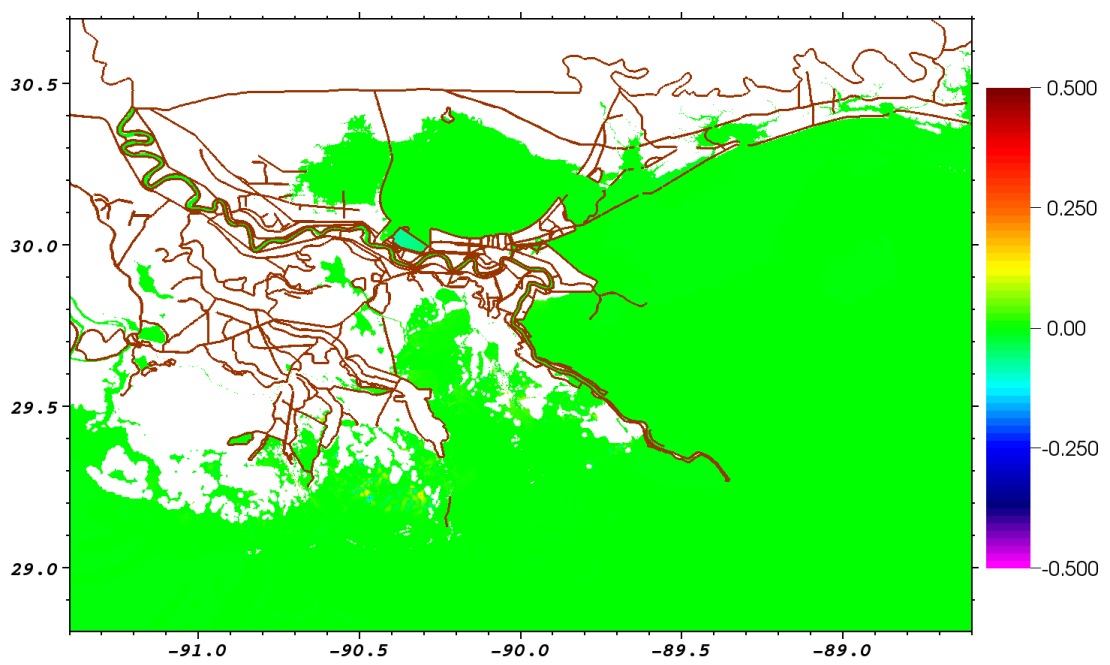

Figure 20: Difference in water surface elevations $(\mathrm{m})$ between $1.0 \mathrm{~s}$ and $0.5 \mathrm{~s}$ time step implicit solutions of the SL15x01 grid. 


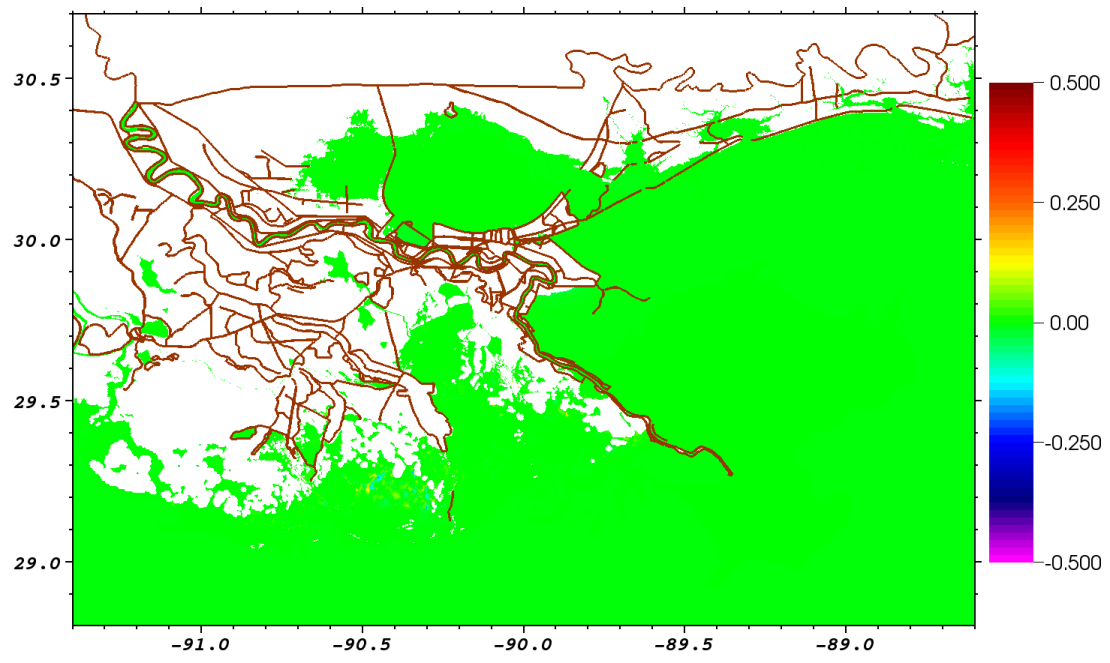

Figure 21: Difference in water surface elevations $(\mathrm{m})$ between 1.0s and $0.5 \mathrm{~s}$ time step size explicit solutions of the SL15x01 grid.

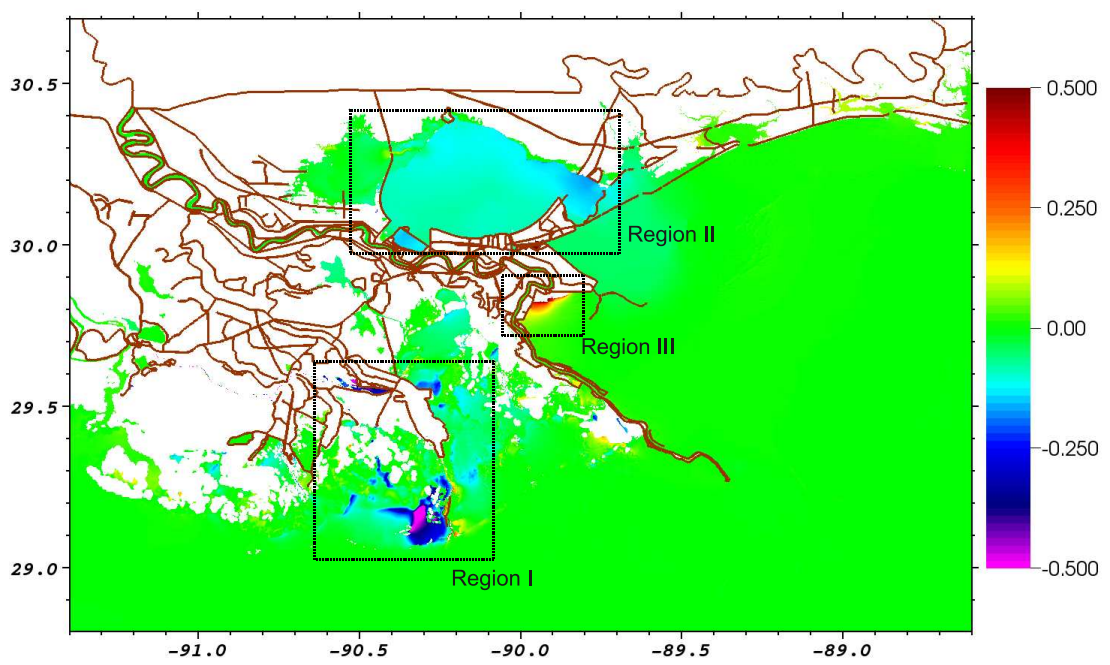

Figure 22: Difference in water surface elevations (m) between the SL15x01 and SL15x04 grids for the implicit solution. 


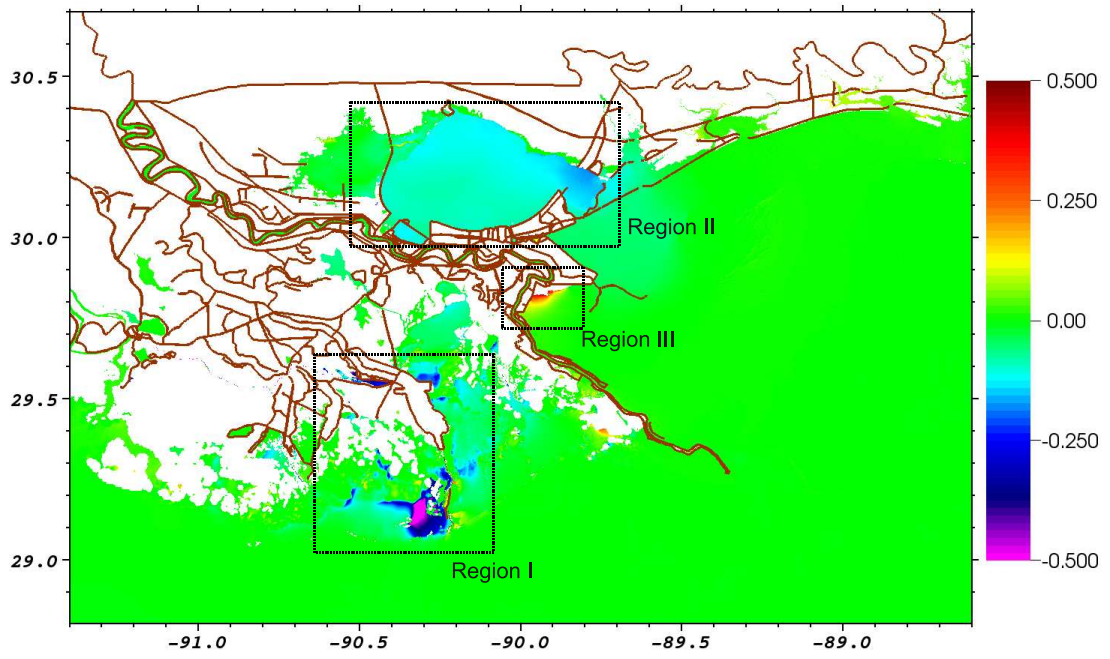

Figure 23: Difference in water surface elevations (m) between the SL15x01 and SL15x04 grid for the explicit solution.

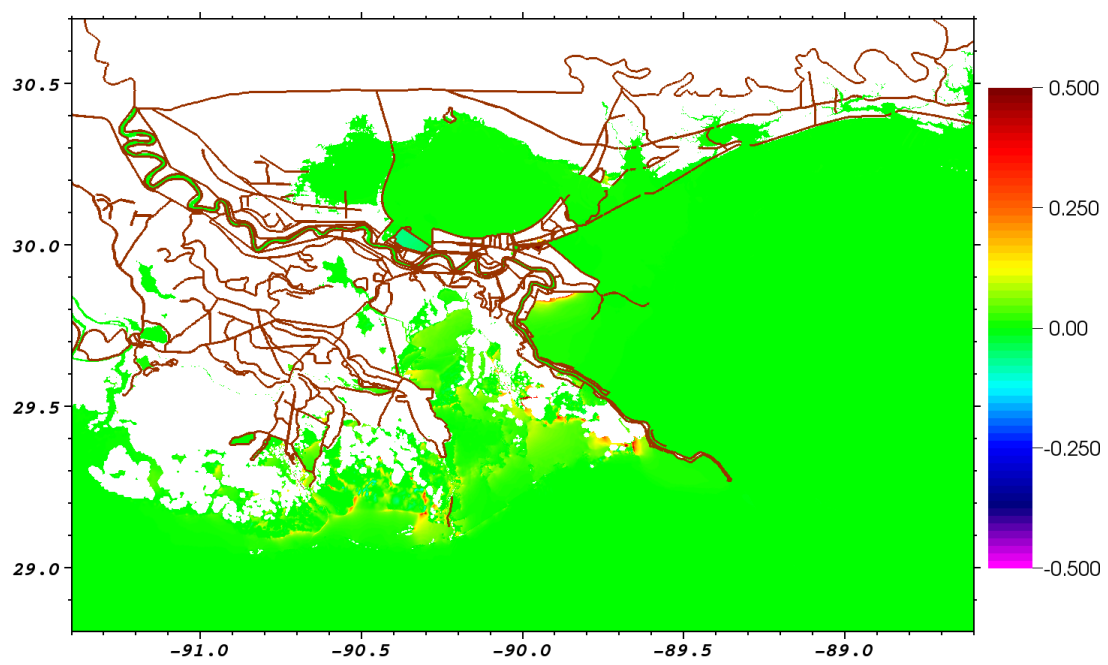

Figure 24: Difference in water surface elevations $(\mathrm{m})$ between the implicit solution and explicit solution of the SL15x01 grid. 


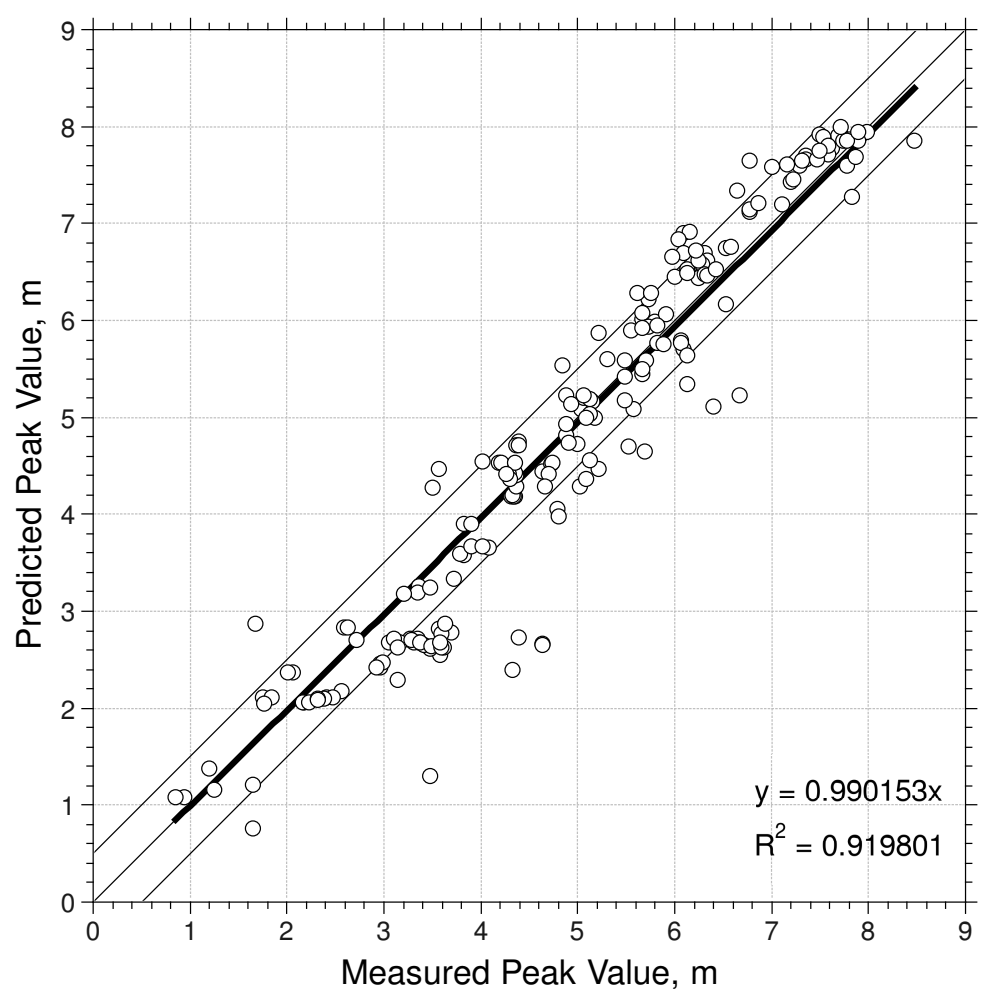

Figure 25: Comparison between observed USACE high water marks and ADCIRC implicit solution of Hurricane Katrina on the SL15x01 grid. 


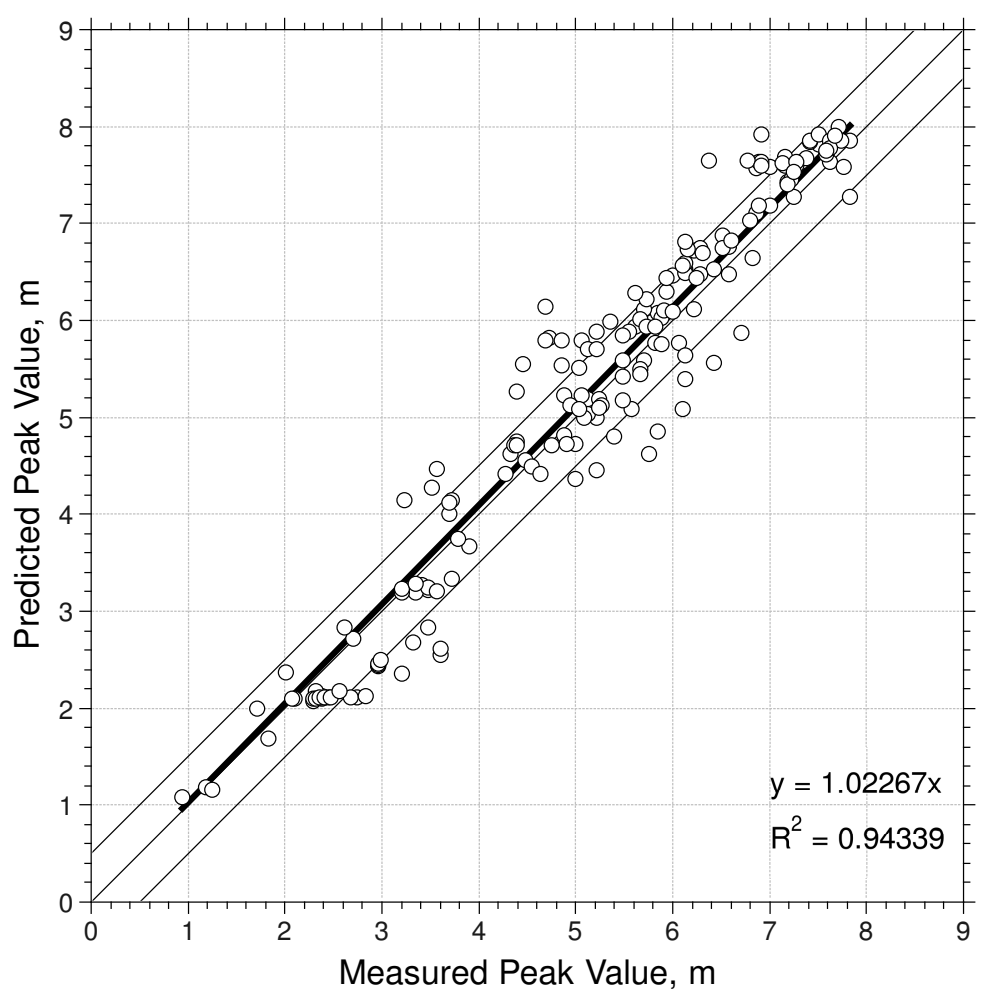

Figure 26: Comparison between observed URS high water marks and ADCIRC implicit solution of Hurricane Katrina on the SL15x01 grid. 

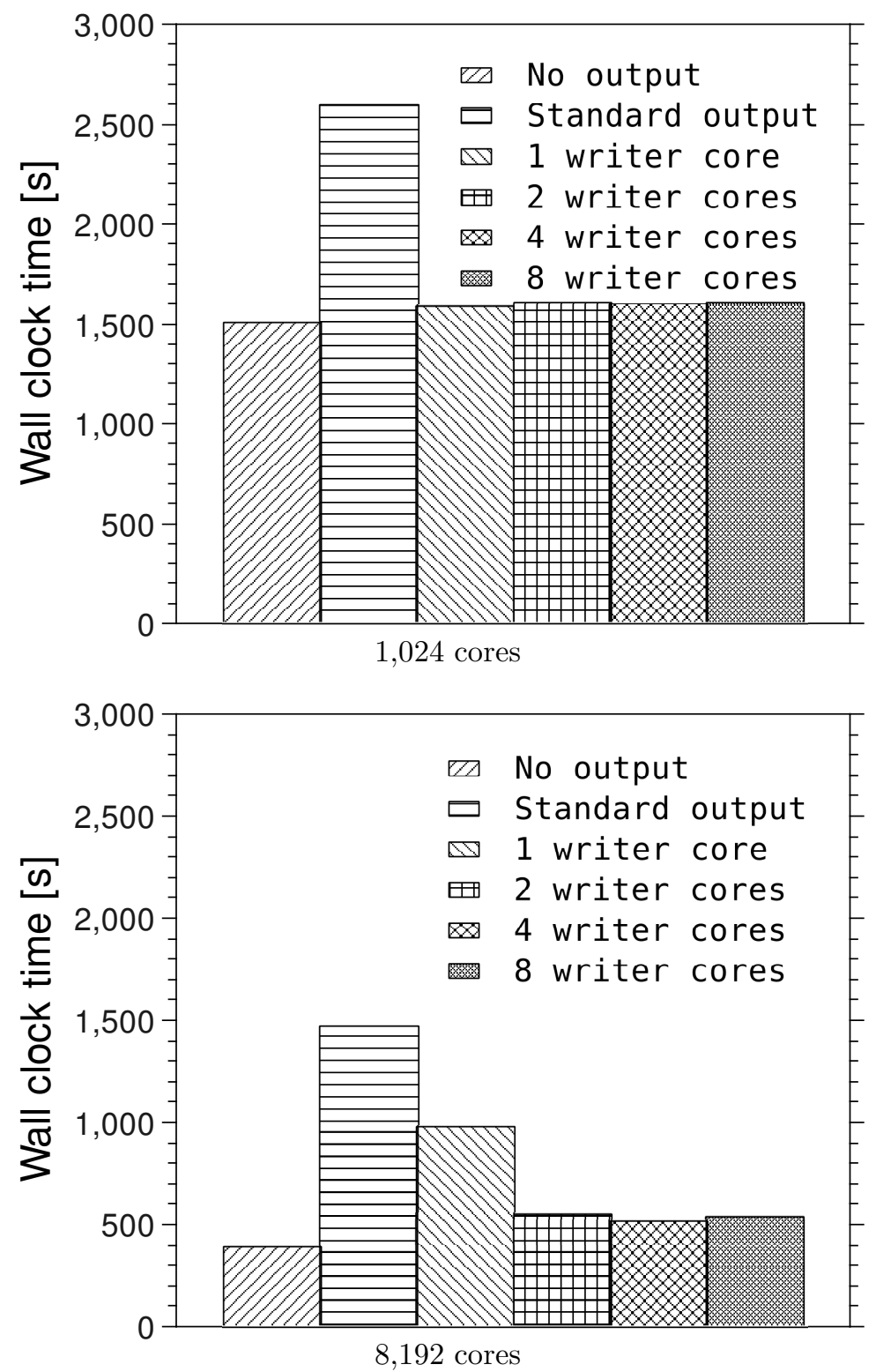

Figure 27: Performance of writing algorithms for the implicit solution of the SL15x04 grid; comparison of no output, standard output, with 1 dedicated writer core, 2 dedicated writer cores, 4 dedicated writer cores, and 8 dedicated writer cores organized in 2 batches. 

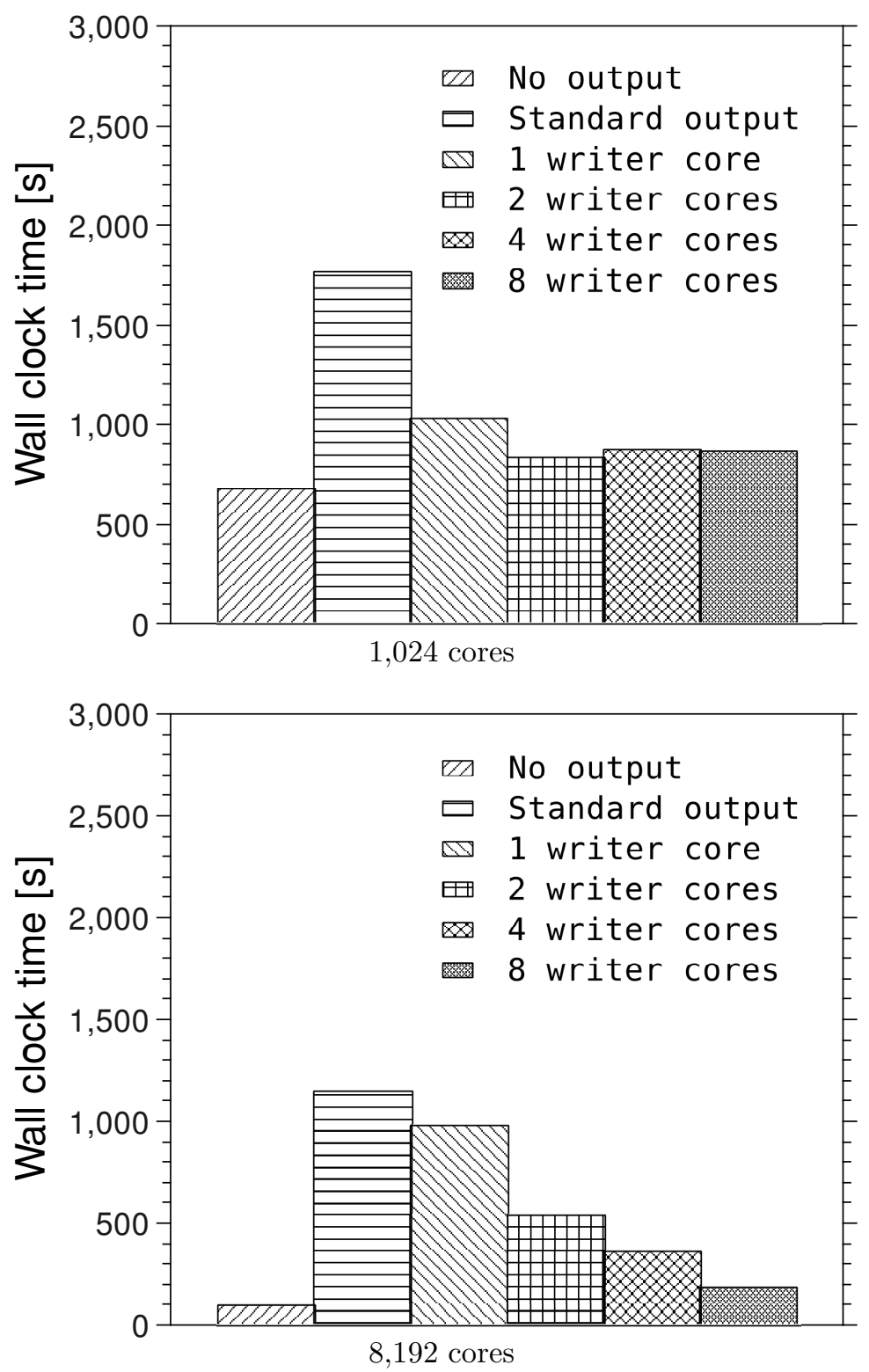

Figure 28: Performance of writing algorithms for the explicit solution of the SL15x04 grid; comparison of no output, standard output, with 1 dedicated writer core, 2 dedicated writer cores, 4 dedicated writer cores, and 8 dedicated writer cores organized in 2 batches. 